\title{
Analysis of sudden variations in photospheric magnetic fields during a large flare and their influences in the solar atmosphere
}

\author{
Brajesh Kumar $^{1}$, A. Raja Bayanna ${ }^{1}$, P. Venkatakrishnan ${ }^{1,2}$ and Shibu K. Mathew ${ }^{1}$ \\ 1 Udaipur Solar Observatory, Physical Research Laboratory, Dewali, Badi Road, Udaipur 313004 , \\ Rajasthan, India; brajesh@prl.res.in \\ 2 Indian Institute of Astrophysics, II Block, Koramangala, Bangalore 560 034, India
}

\begin{abstract}
The solar active region NOAA 11719 produced a large two-ribbon flare on 11 April 2013. We have investigated the sudden variations in the photospheric magnetic fields in this active region during the flare employing the magnetograms obtained in the spectral line Fe I $6173 \AA$ A by the Helioseismic and Magnetic Imager (HMI) onboard Solar Dynamics Observatory $(S D O)$. The analysis of the line-of-sight magnetograms from HMI show sudden and persistent magnetic field changes at different locations of the active region before the onset of the flare and during the flare. The vector magnetic field observations available from HMI also show coincident variations in the total magnetic field strength and its inclination angle at these locations. Using the simultaneous Dopplergrams obtained from HMI, we observe perturbations in the photospheric Doppler signals following the sudden changes in the magnetic fields in the aforementioned locations. The power spectrum analysis of these velocity signals show enhanced acoustic power in these affected locations during the flare as compared to the pre-flare condition. Accompanying these observations, we have also used the near-simultaneous chromospheric observations obtained in the spectral line $\mathrm{H} \alpha 6562.8 \AA$ by the Global Oscillation Network Group (GONG) to study the evolution of flare ribbons and intensity oscillations in the active region. The $\mathrm{H} \alpha$ intensity oscillations also show enhanced oscillatory power during the flare in the aforementioned locations. These results indicate that the transient Lorentz force associated with the sudden changes in the magnetic fields could
\end{abstract}


drive the localized photospheric and chromospheric oscillations, like the flare-induced oscillations in the solar atmosphere.

Key words: Sun: sunspots — Sun: flares — Sun: magnetic fields — Sun: oscillations — Sun: photosphere - Sun: chromosphere

\section{INTRODUCTION}

Solar flares are one of the most catastrophic events taking place in the solar atmosphere. During the flares, the magnetic energy stored in the corona is explosively released on short time-scales (tens of minutes) in the form of thermal radiations in excess of $10^{32} \mathrm{erg}$ and also produces energetic particles moving with very high speeds. The magnetic field configuration in the corona rapidly changes during the flares and the signatures of these appear in the form of the evolution of the photospheric magnetic fields. The changes in the photospheric magnetic fields during flares take place, both on short time-scales of few minutes during the impulsive phase of the flare and longer time-scales of hours covering the phases before and after the flare. The rapid shortterm magnetic field changes (magnetic transients) during flares was first of all reported by Patterson \& Zirin (1981). However, it was interpreted later on (Patterson, 1984; Harvey et al., 1986) that the reported magnetic transients could be attributed to the transient emission of the spectral line being used for measuring the magnetic fields. This interpretation was further established by Qiu \& Gary (2003) through simulations for an observed transient polarity reversal in photospheric magnetic field measurements obtained by Michelson and Doppler Imager (MDI; Scherrer et al., 1995) onboard Solar and Heliospheric Observatory (SOHO; Domingo et al., 1995) spacecraft. On the other hand, Wang (1992) and Wang et al. (1994, 2002) reported rapid and permanent magnetic field changes in flaring active regions using the magnetic field observations at Big Bear Solar Observatory (BBSO). These observations were also confirmed by Kosovichev \& Zharkova (2001) by analyzing the line-of-sight magnetograms obtained from the MDI instrument for an X-class flare on 14 July 2001. Later on, Sudol \& Harvey (2005) and Petrie \& Sudol (2010) extensively analyzed the changes in line-of-sight magnetic fields accompanying several X- and M-class flares. They showed abrupt, significant, and permanent changes in the longitudinal magnetic fields in the flaring active regions. These changes were typically observed to take place in less than 10 minutes with median magnitudes of $100 \mathrm{G}$. This sudden re-configuration of the magnetic fields during the flare produce Lorentz-force-transients in the solar atmosphere and is termed as "magnetic-jerk". Hudson et al. (2008) and Fisher et al. (2012) estimated that the Lorentz force (of the size $\sim 10^{22}$ dynes) associated with the magnetic jerks could be responsible of 
driving localized seismic waves in the solar photosphere. Kumar et al. (2011) found good correspondence between the enhanced localized photospheric velocity oscillations and the sites of magnetic jerks observed in the solar active region NOAA 10930 during an X3.4-class flare on 13 December 2006. Since the magnetic field lines tied to the solar photosphere extends above in the corona, hence it would be important to study the effect of magnetic jerks simultaneously at the solar surface as well as in the higher layers of the solar atmosphere.

In this work, we present a detailed analysis of magnetic and velocity field changes associated with a large two-ribbon flare (of class M6.5) in the solar active region NOAA 11719 on 11 April 2013, using the high-resolution and high-quality co-temporal full-disk photospheric magnetograms and Dopplergrams obtained from the Helioseismic and Magnetic Imager (HMI; Schou et al., 2012) instrument onboard Solar Dynamics Observatory (SDO; Pesnell et al., 2012) spacecraft. Accompanying these observations, we have also used near-simultaneous full-disk chromospheric $\mathrm{H} \alpha$ observations of this flare event obtained from the Global Oscillation Network Group (GONG; Harvey \& GONG Instrument Team, 1995; Harvey et al., 1996) instrument in order to study the morphological and spatial evolution of the flare-ribbons as well as the $\mathrm{H} \alpha$ intensity oscillations during the flare. The motivation of this work is to further investigate the influence of magnetic jerks on the localized photospheric velocity oscillations in the active region during this flare. We also aim to investigate the possible variations in the chromospheric $\mathrm{H} \alpha$ intensity oscillations corresponding to the photospheric locations of the magnetic jerks in the active region during the flare. This would be useful to understand the physical mechanism inter-linking the different layers of the solar atmosphere.

In the following Sections, we describe the observational data, our approach of the data analysis, the results, and the conclusions with a discussion.

\section{THE OBSERVATIONAL DATA}

The solar active region NOAA 11719 appeared on the east limb of the Sun on 5 April 2013 in the northern hemisphere and slowly it evolved into a very complex magnetic region (of class $\beta \gamma \delta$ ). It produced several C- and M-class flares during its passage on the solar disk. On 11 April 2013, a large two-ribbon flare (of class M6.5) occurred around 06:55 UT in this complex active region when it was located at the heliographic coordinates N10E08. The flare produced a high speed Earth-directed Coronal Mass Ejection (CME), type II radio bursts and protons of energy more than $10 \mathrm{MeV}$. Observations related to this interesting flare are available from various space- and ground-based observatories in different wavelengths and energies, viz., SDO, Hinode (Kosugi et al., 2007), Solar TErrestrial RElations Observatory (STEREO; Kaiser et al., 2008), 
Geostationary Operational Environmental Satellite-15 (GOES-15), Nobeyama Radio Polarimeters (NoRP; Nakajima et al., 1985, and references therein), and GONG. In our analysis related to this flare, we employ the photospheric velocity and magnetic field observations obtained by the Helioseismic and Magnetic Imager (HMI) instrument onboard $S D O$, chromospheric $\mathrm{H} \alpha$ observations obtained from the GONG++ telescope (Harvey et al., 2011), and the soft X-ray observations obtained from the GOES-15 satellite during the flare event.

\subsection{SDO/HMI Observations}

The HMI instrument onboard SDO spacecraft provides co-temporal, high quality full-disk photospheric Dopplergrams and line-of-sight magentograms, taken in Fe I $6173 \AA$ line at the cadence of $45 \mathrm{~s}$ with a spatial sampling rate of $\sim 0.5$ arcsec per pixel. HMI derives the line-of-sight velocity and magnetic fields using the Stokes $I$ and $V$ measurements obtained by imaging spectro-polarimetry. The HMI instrument team also provides photospheric vector magnetograms at a cadence of 12 minute using spectropolarimetric measurements of Stokes $I, Q, U$, and $V$. In this study, we have used the tracked grid $\left(\sim 290 \times 193 \operatorname{arcsec}^{2}\right)$ of magnetic maps and Doppler images of the active region NOAA 11719 for the period from 01:00 UT to 10:00 UT on 11 April 2013 to study the flare associated changes in the photospheric magnetic and velocity fields in the active region. The HMI team produces the tracked data of the active region after remapping the region of interest onto heliographic coordinates. In addition to these images, we have also used the continuum images of the active region as observed with HMI to study its morphology during the flare. In the Figure 1, we show the mean images of the active region NOAA 11719 in the continuum, the line-of-sight magnetic fields, and the total Doppler velocity constructed over the time period from 07:00 UT to 08:50 UT on 11 April 2013. It is clearly seen in these images that this active region had a very complex morphology, and hence this is the reason that it produced several flares during its journey through the solar disk.

\subsection{GONG++ H $\alpha$ Observations}

The network of GONG instruments was upgraded to GONG+ (Harvey et al., 1998) by replacing the existing CCD camera of $256 \times 256$ pixels with a new CCD camera having $1024 \times 1024$ pixels for better full-disk spatial resolution in the Dopplergrams, the line-of-sight magnetograms, and the continuum images acquired by this telescope. Later on in mid-2010, GONG+ was upgraded to GONG++ with the addition of $\mathrm{H} \alpha$ image acquisition system to provide a nearly continuous solar activity patrol for use in space weather applica- 
tions (Harvey et al. 2011). Since then, GONG++ network has obtained very useful full-disk chromospheric observations in $\mathrm{H} \alpha 6562.8 \AA$ line at the cadence of one minute and spatial sampling of $\sim 1.0$ arcsec per pixel. The GONG++ instrument nicely covered the full event of this large two-ribbon flare in $\mathrm{H} \alpha$ along with its other regular observations. In the Figure 2, we show the evolution of the flare-ribbons in $\mathrm{H} \alpha$ using the GONG++ observations (negative images). Here, we notice that the flare-ribbons covered the umbra of the sunspot as the flare progressed and also that it was a long duration flare event. We have used these $\mathrm{H} \alpha$ observations simultaneously with the aforementioned HMI observations to study the co-temporal photospheric and chromospheric changes associated with this flare.

\subsection{GOES-15 Soft X-ray Observations}

In order to understand the evolution of flare in the solar environment, we also require the information related to time evolution of high energy radiations from the Sun during the flare. In hard X-ray observations, the Reuven Ramaty High Energy Solar Spectroscopic Imager (RHESSI; Lin et al., 2002) satellite missed to completely cover the impulsive phase of the flare. However, the GOES-15 satellite observations in soft X-ray emissions during the flare are available for nearly the full event. Hence, we have used the disk-integrated soft X-ray data in the 1-8 $\AA$ band from GOES-15 satellite for having temporal information related to the emission of high-energy radiations from the Sun during the flare event. In the Figure 3, we show the temporal

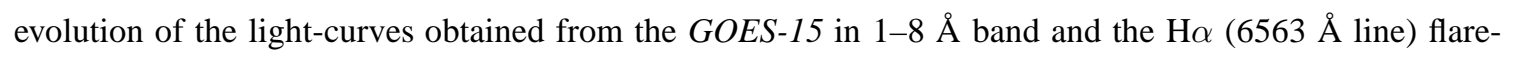
ribbons obtained from the GONG++ instrument. Here, we observe that both the high-energy light-curves peak around 07:15 UT during the flare event. It is also noticed that GOES-15 observations have a gap of nearly 20 minutes during the declining phase of the flare, which would hardly affect our study.

\section{ANALYSIS AND RESULTS}

The chief motivation of this work is to investigate any sudden variations in the line-of-sight photospheric magnetic fields in the localized regions of the active region during the flare and their influence on the localized velocity oscillations on the solar surface. We are also interested to examine the effect of these magnetic jerks in the solar atmosphere. For this purpose, we have employed the co-temporal high resolution photospheric magnetic and velocity field observations from HMI instrument onboard SDO spacecraft and nearly simultaneous chromospheric observations in $\mathrm{H} \alpha$ from GONG++ network. The detail of the data reduction and analysis are presented as follows: 


\subsection{Analysis of Magnetic Field Changes in the Active Region Using HMI Data}

We have analyzed the sequence of tracked grid of photospheric line-of-sight magnetic images from HMI for the period from 06:00 UT to 10:00 UT on 11 April 2013 obtained at the cadence of 45 s, in order to search for the sudden changes in the line-of-sight magnetic fields $\left(B_{l o s}\right)$ in the active region during the flare. The field-of-view of the grid of tracked images is $\sim 290 \times 193 \operatorname{arcsec}^{2}$ and it mostly covers the active region. It is to be noted that the flare started around 06:55 UT and peaked around 07:15 UT on 11 April 2013 as seen in the light-curves of soft X-ray observations from GOES-15 and H $\alpha$ observations from GONG++ (c.f., Figure 3). Hence, the analysis of magnetic fields during the period from 06:00 UT to 10:00 UT would be helpful in the study of magnetic jerks, if any, in the active region during the flare as the aforementioned time period comprises of the observations before, and spanning the flare. We have scanned all the pixels in the field-of-view of the grid of tracked line-of-sight magnetic images for the detection of sudden changes in $B_{l o s}$ in the active region. The following selection criteria have been applied to these magnetic images for identifying such locations in the active region:

(i) Considering that the noise level in the measurement of $B_{l o s}$ of HMI instrument is less than $10 \mathrm{G}$ per pixel, we have considered only those pixels whose average $B_{l o s}$ over a grid of $3 \times 3$ adjoining pixels is more than $\pm 50 \mathrm{G}$ in the given time series.

(ii) The extensive analysis of abrupt changes in longitudinal magnetic fields during several major flares done by Sudol \& Harvey (2005) shows that the typical value of the abrupt changes in $B_{\text {los }}$ is about $90 \mathrm{G}$ observed during those flares. Following this, we have considered only those pixels where the difference between the maximum and minimum of the average $B_{l o s}$ over a grid of $3 \times 3$ adjoining pixels in the time series is more than $100 \mathrm{G}$, separately, for the positive and negative magnetic field regions.

(iii) In order to eliminate the long-term slow variations and trends in the $B_{l o s}$ in the sorted pixels with the aforementioned criteria, their time series of average $B_{l o s}$ over a grid of $3 \times 3$ adjoining pixels is subjected to a $\tilde{\chi}^{2}$ test by applying a linear fit to the aforementioned time series. It is seen that the time series with relatively high $\tilde{\chi}^{2}$ value shows fast variations in $B_{l o s}$. This criterion eliminates the pixels showing slow field evolution with time, and thus we remain with only those time series which have 'step-like changes' in $B_{\text {los }}$ spanning the flare event.

(iv) Sudol \& Harvev (2005) suggest that the abrupt changes in $B_{l o s}$ typically occur in less than 10 minutes while Petrie \& Sudol (2010) suggest that the median duration of abrupt field changes is about 15 minutes. Hence, to further distinguish between normal field evolution and abrupt and persistent changes in the 
$B_{\text {los }}$ spanning the flare event, we have considered only those pixels where more than $100 \mathrm{G}$ change in average $B_{l o s}$ over a grid of $3 \times 3$ adjoining pixels occurs in less than 20 minutes.

Using the above mentioned criteria, we found six locations in the active region where we observe group of pixels showing sudden and persistent changes in the $B_{l o s}$ around the impulsive phase of the flare. These locations are shown as 'K1', 'K2', 'K3', 'K4', 'K5', and 'K6' indicated by yellow circles in the Figure 4. From this figure, we observe that these locations are situated in the vicinity of the $\mathrm{H} \alpha$ flare ribbons as well as away from the flare ribbons. In the left panels of Figure 5, we have plotted the average values of $B_{l o s}$ over a raster of $3 \times 3$ pixels in the aforementioned locations along with the light-curve from GOES-15. A raster of $3 \times 3$ pixels is considered in order to avoid the possible errors in tracking of the grid of images. The centroids of these rasters in the different locations are shown by blue crosses in the Figure 4 . The Carrington heliocentric longitudes and latitudes (in degree) of the aforementioned centroids are as follows: K1(76.91, 12.67), K2(74.06, 9.88), K3(73.49, 12.10), K4(73.64, 14.95), K5(68.75, 14.05), and K6(68.21, 17.26). In the plots of $B_{l o s}$ in the Figure 5, we observe in most of the cases that the value of the longitudinal magnetic fields in the aforementioned locations changes abruptly, then maintains the new value for a period of time that is significantly longer than the duration of the sudden changes in $B_{l o s}$, and then shows unsteady behaviour. Thus, these abrupt changes in $B_{l o s}$ in our observations are "persistent" but not "permanent" changes in longitudinal magnetic fields as reported earlier by Sudol \& Harvey (2005) and Petrie \& Sudol (2010) for other flare events. It is also to be noted that the sudden changes in $B_{l o s}$ appear before the onset of flare as well as during the flare event, and these changes are seen mostly in the weak to moderate magnetic field locations $\left(B_{\text {los }}<500 \mathrm{G}\right)$ in the active region. The amount of change in $B_{l o s}$ ranges between $100 \mathrm{G}$ and $200 \mathrm{G}$ taking place within the time scales of 10 minutes as evident from the plots shown in the Figure 5.

In order to understand the cause behind such variations in $B_{l o s}$, we have studied the morphological evolution of $B_{l o s}$ at the locations 'K1', 'K2', 'K3', 'K4', 'K5', and 'K6' in the active region during the flare. In the Figure 6, we show the mosaic of the time evolution of $B_{l o s}$ over a grid of $15 \times 15$ pixels containing the raster of $3 \times 3$ pixels at its center (enclosed by white box) corresponding to the locations $\mathrm{K} 1(76.91$, 12.67), $\mathrm{K} 2(74.06,9.88), \mathrm{K} 3(73.49,12.10), \mathrm{K} 4(73.64,14.95), \mathrm{K} 5(68.75,14.05)$, and $\mathrm{K} 6(68.21,17.26)$ in the active region for the period from 06:30 UT to 08:18 UT at the time interval of twelve minutes. The time duration from 06:30 UT to 08:18 UT covers the pre-flare phase and the flare phase. In this illustration of the morphological evolution of $B_{l o s}$, we observe fast changes in the magnetic concentrations in these affected locations. We conjecture that the sudden changes in the $B_{l o s}$ observed at the aforementioned locations could 
be due to the process of fast re-organization of the coronal magnetic fields during the flare, thereby producing fast changes in the directions of the photospheric magnetic fields.

We have also used the tracked vector magnetic field data available from HMI at the cadence of twelve minute for the period from 06:00 UT to 10:00 UT on 11 April 2013, in order to study the variations in the total magnetic field strength $\left(B_{0}\right)$ and the inclination angle $(\gamma)$ of the magnetic field lines at the locations of sudden changes seen in $B_{l o s}$ in the active region. These data are co-aligned with the main grid of $B_{l o s}$ images $\left(\sim 290 \times 193 \operatorname{arcsec}^{2}\right)$ for estimating $B_{0}$ and $\gamma$ at the affected locations. In the Figure 7, we show the plots of $B_{0}$ and $\gamma$ averaged over the same rasters of $3 \times 3$ pixels at the locations $\mathrm{K} 1(76.91,12.67), \mathrm{K} 2(74.06$, 9.88), $\mathrm{K} 3(73.49,12.10), \mathrm{K} 4(73.64,14.95), \mathrm{K} 5(68.75,14.05)$, and $\mathrm{K} 6(68.21,17.26)$ in the active region as considered for $B_{l o s}$ (c.f., Figure 5) for the period from 06:00 UT to 10:00 UT on 11 April 2013. Here, we notice that both $B_{0}$ and $\gamma$ show sudden and persistent variations around the time of the sudden changes seen in the $B_{l o s}$. This implies that the sudden and persistent changes seen in the $B_{l o s}$ are due to the simultaneous sudden changes in $B_{0}$ and $\gamma$ at the locations ' $\mathrm{K} 1$ ', 'K2', 'K3', 'K4', 'K5', and ' $\mathrm{K} 6$ ' in the active region.

These sudden changes in the magnetic fields at the aforementioned locations would produce Lorentzforce-transients or the "magnetic-jerk" in these affected areas.

\subsection{Analysis of Velocity Field Changes in the Active Region Using HMI Data}

The co-temporal full-disk photospheric Dopplergrams and line-of-sight magnetograms obtained by HMI provides the excellent opportunity to examine the changes in the velocity signals associated with the sites of magnetic jerks ('K1', 'K2', 'K3', 'K4', 'K5', and 'K6') in the active region. Therefore, we have analyzed the sequence of tracked grid $\left(\sim 290 \times 193 \mathrm{arcsec}^{2}\right)$ of Doppler velocity images from HMI for the period from 06:00 UT to 10:00 UT on 11 April 2013 at the cadence of 45 s. In our analysis, we have studied the temporal evolution of the mean velocity signals from the aforementioned images over the same grids of $3 \times 3$ pixels at the centroid of the sites of magnetic jerks in the active region with the Carrington heliocentric longitudes and latitudes (in degree) as follows: K1(76.91, 12.67), K2(74.06, 9.88), K3(73.49, 12.10), K4(73.64, 14.95), $\mathrm{K} 5(68.75,14.05)$, and K6(68.21, 17.26). In the right panels of the Figure 5, we have plotted these mean line-of-sight velocity signals for the period from 06:00 UT to 10:00 UT on 11 April 2013 after removing the large background gradient in the observational data appearing mainly due to the orbital velocity of the satellite. Here, we observe significant perturbations in the photospheric Doppler signals following the epochs of the sudden and persistent changes seen in $B_{l o s}$ in the aforementioned locations of the active region during the flare. The signature of enhancements seen in the velocity signals associated with these sites of 


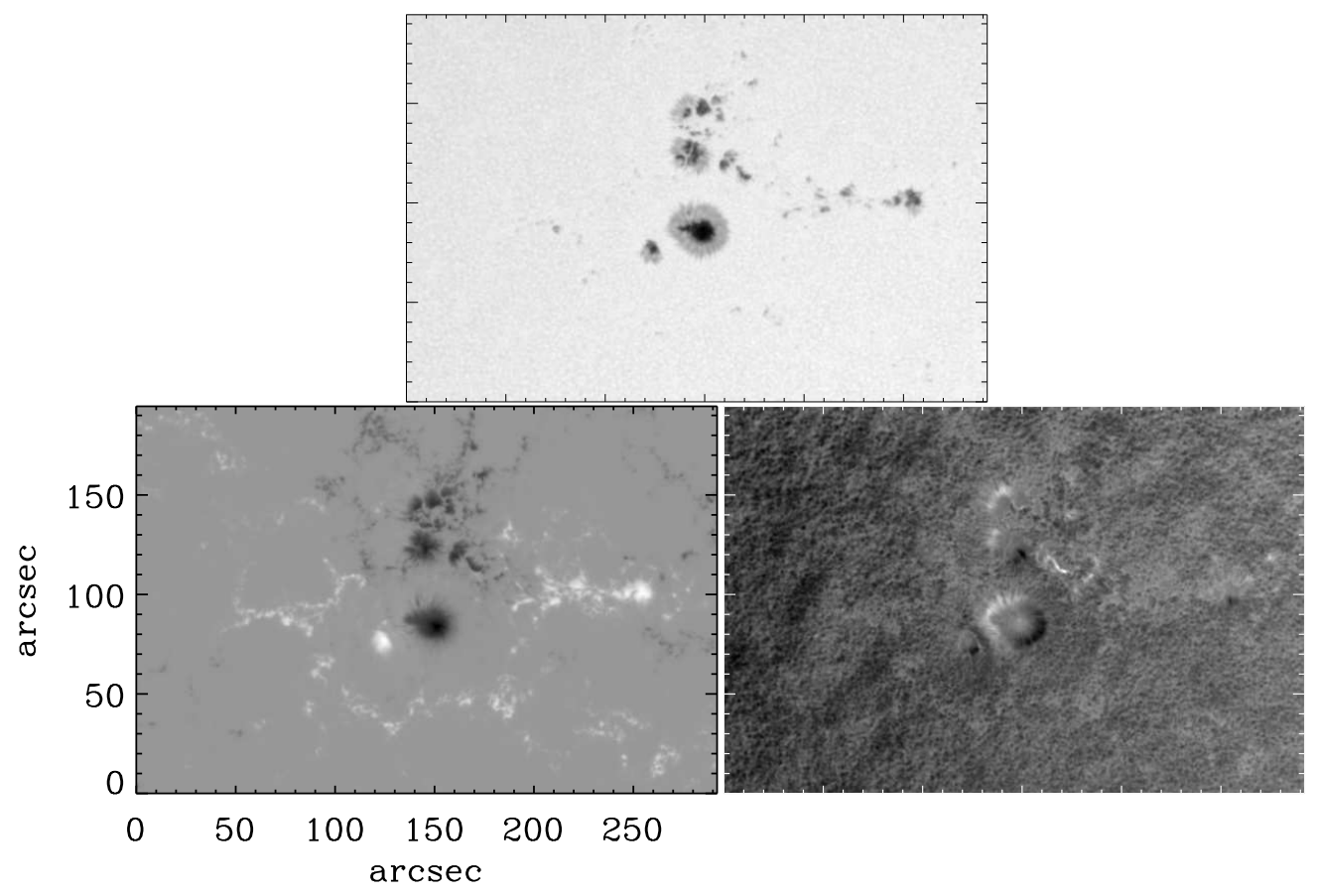

Fig. 1 The images of the active region NOAA 11719 in the continuum intensity (top panel), the line-of-sight photospheric magnetic fields (bottom left panel), and the photospheric Doppler velocity (bottom right panel) as observed with HMI instrument onboard SDO spacecraft. All the images shown here are the mean images constructed over the time interval 07:00-08:50 UT spanning the flare on 11 April 2013.

magnetic jerks motivated us to peform power spectrum analysis of these velocity variations during the preflare phase (01:00-05:00 UT) and the flare phase (06:00-10:00 UT) covering the magnetic jerks. Therefore, we applied Fourier transform to these line-of-sight velocity signals from each individual pixels in the grid of $3 \times 3$ pixels and then an average power spectrum of velocity oscillations is constructed for each of the aforementioned rasters in the locations 'K1', 'K2', 'K3', 'K4', 'K5', and 'K6' in the active region for the pre-flare phase and the flare phase. In the Figure 8, we show in the solid black lines the average power spectra of velocity oscillations for the aforementioned locations in the active region for pre-flare and flare conditions. A smoothing fit (Savitszky-Golay Fit; Press et al. (1992)) is applied (shown in the solid red lines) to these original power spectra for estimating the power envelopes. 


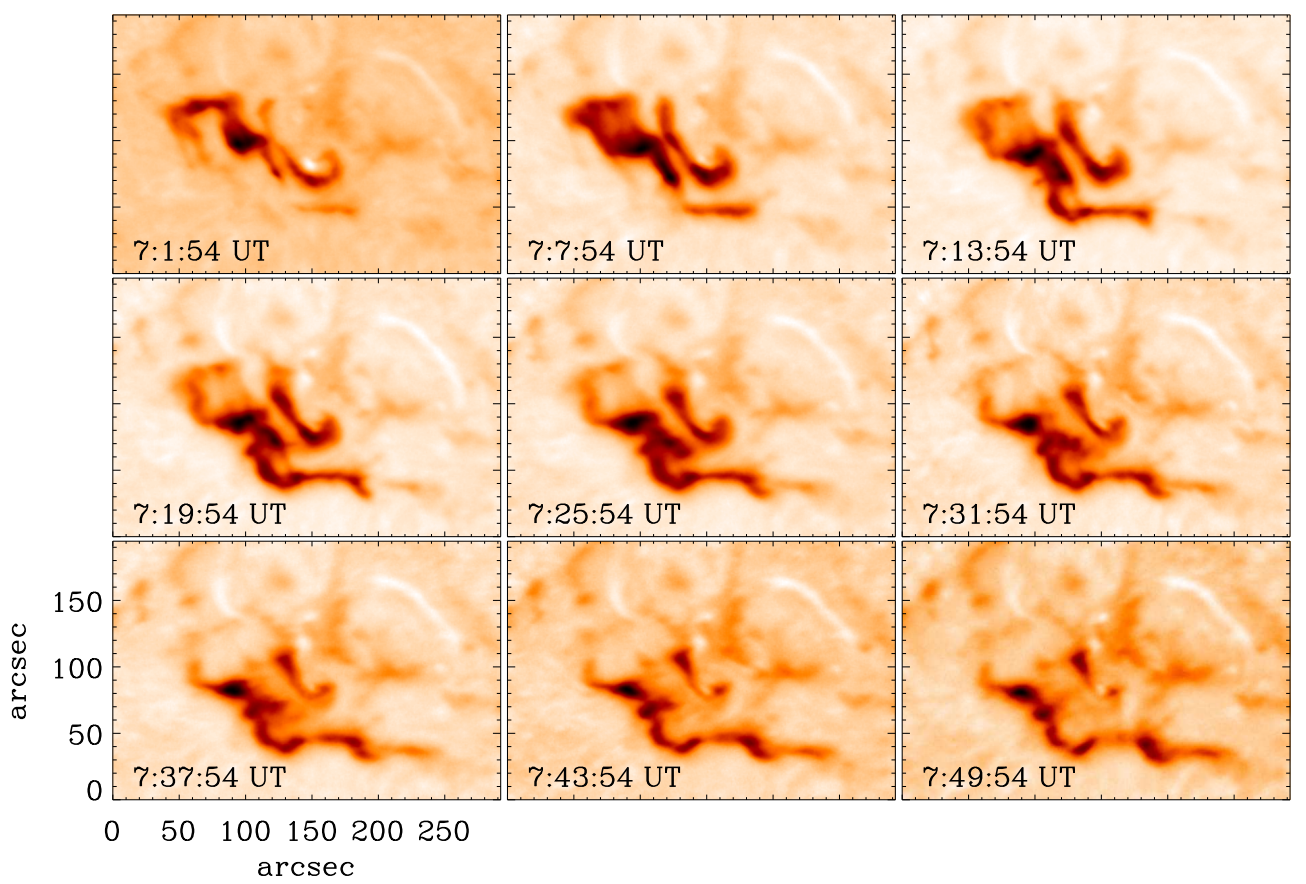

Fig. 2 Mosaic of images showing the evolution of the flare-ribbons in $\mathrm{H} \alpha 6563 \AA$ line during the period 07:01:54-07:49:54 UT on 11 April 2015 using the GONG++ observations. Here, we notice that this was a two-ribbon flare and the flare-ribbons separated apart and covered the umbra of the sunspot as the flare progressed. The images are shown in negative for better appearance of the morphology of the flare-ribbons.

It is evident from these velocity power spectra that there is enhancement of power of velocity oscillations in the $2-5 \mathrm{mHz}$ band in the aforementioned locations during the flare as compared to the pre-flare condition. In this context, it is worthy mentioning that if the magnitude of the magnetic flux of the selected region changes, this would affect the amplitude of velocity oscillations in those regions leading to a change in the power of acoustic spectra. Thus, if there is a gross decrease in the magnitude of magnetic flux in the selected region during the flare, the velocity power spectra would show an increase of power with respect to that in the pre-flare condition and vice-versa. Hence, the method of comparing pre-flare velocity power spectrum with respect to that estimated during the flare may lead to erroneous interpretations. Therefore, we have checked the magnitude of $B_{l o s}$ averaged over the aforementioned grids of $3 \times 3$ pixels in the locations ' $\mathrm{K} 1$ ', 'K2', 'K3', 'K4', 'K5', and 'K6' during the pre-flare and flare epochs considered in our analysis. It is seen 


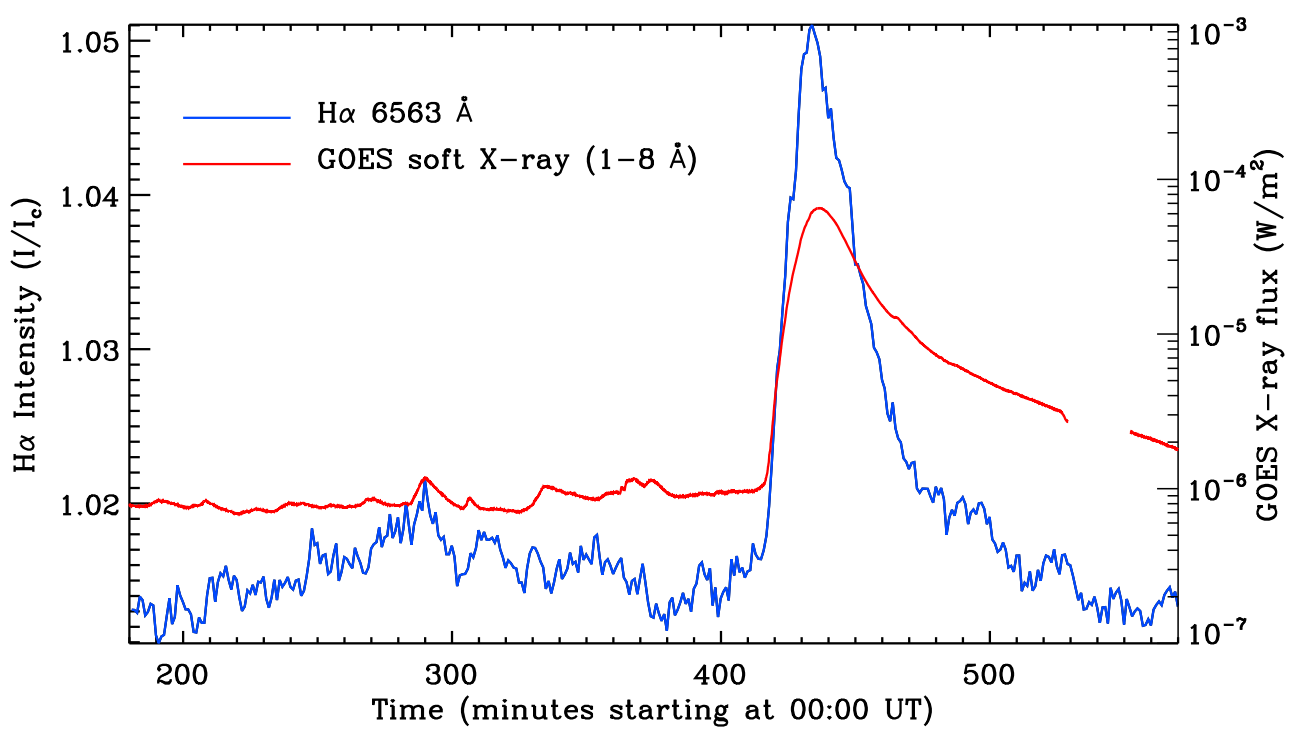

Fig. 3 Plots showing the the temporal evolution of the light-curves obtained from the GOES-15 in 1-8 $\AA$ band and the $\mathrm{H} \alpha(6562.8 \AA$ line) flare-ribbons obtained from the GONG++ instrument during the period 03:00-09:30 UT on 11 April 2013. Here, we observe that both the high-energy light-curves peak around 07:15 UT during the flare event. It is also noticed that GOES-15 observations have a gap of nearly 20 minutes during the declining phase of the flare.

that there is a gross increase in the magnitude of average $B_{l o s}$ in the locations ' $\mathrm{K} 1$ ', and ' $\mathrm{K} 3$ ' while there is a gross decrease in the magnitude of average $B_{l o s}$ in the locations ' $\mathrm{K} 2$ ', ' $\mathrm{K} 4$ ', ' $\mathrm{K} 5$ ', and 'K6' during the flare phase as compared to the pre-flare phase. Also, it is to be noted that ' $\mathrm{K} 1$ ' and ' $\mathrm{K} 3$ ' are much away from the flare-ribbons. However, we observe an enhancement in the power of velocity oscillations in all the locations 'K1', 'K2', 'K3', 'K4', 'K5', and 'K6'. This implies that the effect of "magnetic-jerk” dominates the effect of the normal evolution of magnetic concentrations in the aforementioned locations concerning the enhancement of power of the velocity oscillations in these identified locations.

In order to further verify our aforementioned observations and the interpretations, we have done similar analysis for some other locations in and around this active region for the pre-flare phase and the flare phase. For this purpose, we have considered two locations ('M1', and 'M2') in the active region which show gradual evolution of $B_{\text {los }}$ during the flare, and two locations ('Q1', and 'Q2') in the quiet Sun. The centroids of these locations are shown by yellow crosses within blue circles in the Figure 4 . The Carrington heliocentric longi- 
tudes and latitudes (in degree) of the aforementioned centroids are as follows: M1(73.76, 10.96), M2(80.18, 9.61), Q1 (67.13, 4.78), and Q2 (81.56, 14.05). In the Figure 9, we have plotted the mean $B_{l o s}$ (left panels) and the mean line-of-sight velocity signals (right panels) after removing the large background gradient over a raster of $3 \times 3$ pixels for the aforementioned kernels during the period from 06:00 UT to 10:00 UT on 11 April 2013. In the case of 'M1', we observe a gross decrease of $\sim 200 \mathrm{G}$ in the $B_{\text {los }}$ whereas 'M2' shows a gross decrease of $\sim 500 \mathrm{G}$ in the $B_{l o s}$ during the flare. Here, we do not notice any significant perturbations in the photospheric Doppler signals during the flare in these locations of the active region. In the quiet locations 'Q1', and 'Q2', the variations in the mean $B_{l o s}$ are within the noise level of the instrument ( 10 G) while the mean line-of-sight velocity signals are dominant and show normal evolution during the flare. In the Figure 10, we show the average power spectra of velocity oscillations in these locations for pre-flare phase (01:00-05:00 UT) and the flare phase (06:00-10:00 UT). In the case of 'M1', and 'M2', we notice from these velocity power spectra that there is enhancement of power of velocity oscillations in the $2-5 \mathrm{mHz}$ band during the flare as compared to the pre-flare condition. We also observe that the enhancement in oscillatory power at the location 'M2' is more as compared to that in the location 'M1'. Here, it is worthy mentioning that during the flare the gross decrease in $B_{l o s}$ is more at 'M2' as compared to that in 'M1' and hence this has resulted into relatively more enhancement of oscillatory power at 'M2'. In the case of 'Q1', and 'Q2', we do not observe any significant variations in the power of the velocity oscillations during the flare as compared to the pre-flare condition. These results show that the evolution of magnetic concentrations in the locations 'M1', and 'M2' is controlling the enhancement of the power of the velocity oscillations in these locations during the flare, while 'Q1', and 'Q2' remain unaffected being in the quiet Sun. However, as we have discussed earlier this assumption is not applicable at the sites of the magnetic jerks in the active region during the flare. We have observed enhancements in the acoustic power at the locations of magnetic jerks irrespective of the increase or decrease of $B_{l o s}$ at these locations during the flare with respect to the pre-flare condition. It is also worthy to note that the effect of magnetic jerks is more pronounced on the power of acoustic oscillations as compared to that due to the normal variations in the $B_{l o s}$ at the locations ' $\mathrm{M} 1$ ', and 'M2'.

The another important point to mention is that we do not observe any instantaneous large velocity signal appearing right at the time of the impulsive phase of the flare as has been seen in the active regions during several major X-class flares. Such large spiky velocity and magnetic signals may also appear due to distortions in the line profile in the flaring regions. In our case, the identified locations are in the vicinity of the flare-ribbons and also away from the sites of these ribbons. We observe relatively enhanced photospheric 
velocity signals following the phenomena of sudden and persistent changes seen in $B_{l o s}$ in the active region and this is the reason why these lead to enhancement in the gross power of the acoustic spectra for the flare phase covering the magnetic jerks, as compared to the pre-flare phase.

\subsection{Analysis of $\mathrm{H} \alpha$ Intensity Oscillations in the Active Region using GONG++ Data}

We have near-simultaneous sequence of chromospheric observations consisting of full-disk $\mathrm{H} \alpha$ filtergrams available from GONG++ along with the above magnetic and velocity field observations from HMI. The availability of these $\mathrm{H} \alpha$ observations from GONG++ provides the opportunity to study the possible changes in the $\mathrm{H} \alpha$ intensity oscillations driven by the aforementioned "magnetic-jerk" in the active region appearing during the flare. We have reasonably good quality full-disk $\mathrm{H} \alpha$ images available from GONG++ instrument for the period from 03:01 UT to 09:31 UT on 11 April 2013 at a cadence of one minute. The sequence of these every minute $\mathrm{H} \alpha$ images are derotated and registered with respect to the image taken at 03:01 UT with an accuracy of $0.1 \mathrm{arcsec}$. A grid of the size $\sim 290 \times 193 \operatorname{arcsec}^{2}$ consisting of the active region is selected from each of the derotated and registered sequence of the full-disk $\mathrm{H} \alpha$ images. The sequence of these images are aligned with respect to the image at 03:01 UT using Fast Fourier transform based cross-correlation algorithm with an accuracy of 0.1 arcsec. These registered images are re-scaled to the spatial scale of $0.5 \operatorname{arcsec}$ per pixel in order to match with the pixel scale of HMI data. The re-scaled image sequence is then co-aligned with respect to the field-of-view of the grid of magnetic and velocity images from HMI used in our analysis. The sequence of these co-aligned grid of $\mathrm{H} \alpha$ images are segregated into two parts for our analysis: the pre-flare phase (03:01-06:01 UT) and the flare phase (06:31-09:31 UT). Now, it would be reasonable to consider that the locations of the "magnetic-jerk" seen in the photosphere would not be the same in the chromosphere due to the inclination of the magnetic field lines. Hence, we have scanned through the pixels around the centroids of the grid of $3 \times 3$ pixels in the locations ' $\mathrm{K} 1$ ', 'K2', 'K3', 'K4', 'K5', and 'K6' as considered in the case of HMI magnetic and velocity images in order to find the best locations showing any "magnetic-jerk" driven chromospheric oscillations in the active region during the flare. Following this, we found such locations in these $\mathrm{H} \alpha$ images which show enhancement of power in intensity oscillations during the flare and are situated little away (within 2 arc-sec) from the aforementioned centroids of the sites of magnetic jerks in the photospheric magnetograms in the locations 'K1', 'K2', 'K3', 'K4', 'K5', and 'K6'. However, these new locations seen in $\mathrm{H} \alpha$ images do not exactly match with the deviations as estimated $(\approx h \cdot \tan (\gamma) ; h=$ difference in the average formation heights of the lines) from the inclination angles $(\gamma)$ available from the photospheric vector magnetograms from HMI. The most important reason for this mis- 
match could be the canopy effect in the chromosphere, where the magnetic field lines spread out and become more tilted in general than in the photosphere. However, the other probable sources of errors could be as follows: (i) the seeing effects in the $\mathrm{H} \alpha$ ground-based observations obtained from GONG++ as compared with the space-based observations with HMI, (ii) the errors in the co-alignment of the images from the two different instruments with different spatial resolutions, and (iii) the difference in the available observational cadence of $B_{\text {los }}$ ( $\left.45 \mathrm{~s}\right)$ and $\gamma(12$ minute). In the Figure 11, we show the evolution of normalized $\mathrm{H} \alpha$ light curves averaged over a grid of $3 \times 3$ pixels in the new locations within 'K1', 'K2', 'K3', 'K4', 'K5', and 'K6' in the active region during the phases of pre-flare and flare conditions. These $\mathrm{H} \alpha$ light curves exhibit quasiperiodic intensity variations which are similar to the observations of Wang et al. (2000) for a C5.7 flare that ocurred in the solar active region NOAA 8673 on 23 August 1999. Wang et al. (2000) used high-resolution $\mathrm{H} \alpha$ observations of this C-class flare obtained at BBSO. Resembling the velocity power spectrum, we have estimated the Fourier power spectrum of the $\mathrm{H} \alpha$ intensity oscillations in each of the individual pixels in the new grids of $3 \times 3$ pixels in the aforementioned locations and constructed average power spectra for the pre-flare and flare phases (c.f., Figure 12). It is evident from these power spectra that there is enhancement of power in chromospheric intensity oscillations in these locations during the flare as compared to the pre-flare condition.

\section{DISCUSSION AND CONCLUSIONS}

We have investigated the photospheric magnetic and velocity field changes in the active region NOAA 11719 during a large two-ribbon flare (of class M6.5) that occurred on 11 April 2013, using the high-quality observations obtained from the HMI instrument onboard SDO spacecraft. Accompanying these photospheric observations, we have also analyzed the near-simultaneous chromospheric $\mathrm{H} \alpha$ observations from the GONG++ instrument in order to understand any possible inter-linked physical process taking place between the different layers in the solar atmosphere. The chief findings of our investigations and the interpretations of our results are as follows:

i. The analysis of the line-of-sight magnetic fields $\left(B_{l o s}\right)$ from HMI shows sudden and persistent magnetic field changes at different locations of the active region during the flare. These sites ('K1', 'K2', 'K3', ' $\mathrm{K} 4$ ', 'K5', and 'K6') are located in the vicinity of the $\mathrm{H} \alpha$ flare ribbons, as well as away from the flare ribbons in the active region. We also observe that these abrupt changes in $\left(B_{l o s}\right)$ appear before the onset of flare, as well as during the flare and are seen mostly in the weak to moderate magnetic field concentrations in the active region. Burtseva et al. (2015) analyzed several X-class flares and have reported abrupt magnetic 
field changes which take place before or around the start time of the flare. They have also shown that these field changes and the foot-points of the hard X-ray emission during the flare are not always co-spatial. Our results show that the amount of change in $B_{l o s}$ is between $100 \mathrm{G}$ and $200 \mathrm{G}$ taking place within the time scales of 10 minutes, which are similar to the findings of Sudol \& Harvey (2005) and Petrie \& Sudol (2010). However, the abrupt changes in $B_{l o s}$ in our observations are "persistent" but not "permanent" changes in longitudinal magnetic fields as have been found by Sudol \& Harvey (2005) and Petrie \& Sudol (2010) for other flare events. From our analysis related to the morphological evolution of $B_{l o s}$ at these locations, we conjecture that the sudden changes in the $B_{l o s}$ observed at these locations could be due to the process of fast re-organization of the cornal magnetic fields during the flare resulting into fast changes in the directions of photospheric vector fields. We have also analyzed the vector magnetic field data available from HMI which show that the sudden changes seen in the $B_{l o s}$ are accompanied with the coincident sudden changes in $B_{0}$ and $\gamma$ at these locations in the active region. There have been always a concern regarding the distortions in the line-profile affecting the observations of magnetic and velocity fields in the active region during the impulsive phase of the large flares (Qiu \& Gary, 2003). However, this speculation could be applicable for the locations situated within the flare-ribbons or the hard X-ray foot-points. In such cases, large short-lived changes appear in the magnetic and velocity fields during the flare. In our case, some of the affected locations ('K1', 'K3', and 'K4') are away from the $\mathrm{H} \alpha$ flare ribbons and hence these are free from the possible effect of distortions in the line profile. On the other hand, for the locations in the vicinity of $\mathrm{H} \alpha$ flare ribbons ('K2', 'K5', and 'K6'), we do not observe any large spiky signals appearing for short-times in the $B_{\text {los }}$ and Doppler velocity signals at these locations. It is also important to note that the profiles of $B_{l o s}$ and Doppler velocity for all the locations 'K1', 'K2', 'K3', 'K4', 'K5', and 'K6' appear similar in nature. Hence, we suggest that the sudden changes appearing in $B_{l o s}$ and the subsequent perturbations in the Doppler velocity signals in the aforementioned locations are real changes happening in the active region during the flare.

ii. It is believed that the Lorentz-force-transients associated with the "magnetic-jerk" can drive localized seismic waves in the solar photosphere (Hudson et al., 2008; Fisher et al., 2012). Hence, we have analyzed the HMI Dopplergrams for the photospheric velocity signals in the locations 'K1', 'K2', 'K3', 'K4', 'K5', and 'K6' for the epochs, before and spanning the flare. For this purpose, we have estimated Fourier power spectrum of these velocity oscillations in the aforementioned locations. It is observed that the power of velocity oscillations is enhanced in the locations of magnetic jerks spanning the flare as compared to the pre-flare condition. This enhancement is found for all the sites of magnetic jerks in the active region during the flare. There are other physical processes responsible for driving oscillations in the solar 
atmosphere during the flare, mainly the chromospheric shocks propagating through the photosphere into the solar interior (Fisher et al., 1985) or the high-energy particle beam impinging on the solar photosphere (Venkatakrishnan et al., 2008, and references therein). However, this is applicable for the situation when the affected areas are within the hard X-ray foot-points or $\mathrm{H} \alpha$ flare kernels. In our case, the affected locations are situated in the vicinity of the flare ribbons and also much away from the flare ribbons in the active region, hence this indicates that magnetic jerks have certainly driven these localized photospheric velocity oscillations. A general estimate is that the transient Lorentz forces of the size $\sim 10^{22}$ dynes (Hudson et al., 2008; Fisher et al., 2012) associated with these magnetic jerks could be responsible for driving localized seismic waves in the solar photosphere. Following Fisher et al. (2012), we estimate the approximate transient Lorentz force $\left(B_{l o s} \cdot \delta B_{l o s} \cdot \mathrm{A} / 4 \pi\right)$ corresponding to the sites of the magnetic jerks in the active region during this flare event. In our case, the mean value of the line-of-sight magnetic field changes $\left(\delta B_{l o s}\right)$ is $\sim 150 \mathrm{G}$ and the mean value of $B_{l o s}$ at these locations is $\sim 300 \mathrm{G}$ over an area (A) of $\sim 10^{16} \mathrm{~cm}^{2}$ as obtained from HMI magnetograms in the locations of the magnetic jerks. Hence, the approximate estimate of the transient Lorentz force at these sites of magnetic jerks turns out to be $\sim 10^{19}$ dynes. This is a lower available budget of the transient Lorentz force as compared to the required size of the force for driving the localized seismic waves. It is to be noted that the expression of Fisher et al. (2012) for estimating the transient Lorentz force is applicable for strong magnetic field regions ( $\geq 1 \mathrm{kG}$ optimally at $\tau=1$ opacity layer at the wavelength $5000 \AA$ ) and also approximated under some theoretical assumptions (For a detailed discussion, please refer to Petrie, 2014). In our case, the sites of magnetic jerks are located in weak to moderate field regions $(\leq 500 \mathrm{G})$ and also our estimate suffers from the non-availability of all the vector components simultaneously. Hence, the above constraints could be the reason for the deficit budget of transient force appearing in our estimation, by using the expression of Fisher et al. (2012) under approximations. However, we do observe enhancements in the power of velocity oscillations in the locations of the magnetic jerks in the active region during the flare. In our observations, the average change in the amplitude of localized velocity oscillations $(\Delta v)$ associated with the magnetic jerks is $\sim 200 \mathrm{~m} / \mathrm{s}$. Hence, the estimate of the transient force required to drive these acosutic oscillations could be $\approx \rho \cdot\left(\Delta v^{2}\right)$.A, where ' $\rho$ ' is the mean density of the solar photosphere $\left(\sim 2 \times 10^{-7} \mathrm{gcm}^{-3}\right)$. This yields a required budget of the driving force of $\sim 8 \times 10^{17}$ dynes against the available budget of the transient force of $\sim 10^{19}$ dynes as estimated from the magnetic jerks. Thus, from the aforementioned calculation it appears that the magnetic jerks at the locations 'K1', 'K2', 'K3', 'K4', 'K5', and 'K6' are capable of driving the localized photospheric acoustic oscillations. 
iii. Using the $\mathrm{H} \alpha$ chromospheric observations of this flare from GONG++ instrument, we have analyzed the changes in the $\mathrm{H} \alpha$ intensity oscillations in the corresponding locations of the magnetic-jerks in the active region during the flare. The motivation of this analysis is to study the possible inter-linking physical processes between the different layers of the solar atmosphere. For this purpose, we have applied Fourier transform to the $\mathrm{H} \alpha$ intensity oscillations in these affected locations for the epochs, before and spanning the flare. It is observed that there is enhancement of the power of $\mathrm{H} \alpha$ intensity oscillations in these locations during the flare. The power enhancement is seen at different frequencies for the kernels ' $\mathrm{K} 1$ ', 'K2', 'K3', 'K4', 'K5', and 'K6'. These findings are similar to the results of Wang et al. (2000) in the case of flare driven $\mathrm{H} \alpha$ intensity oscillations. In our case, some of the locations are in the vicinity of the flare-loops and hence the flare would have mainly contributed for the enhancement of the chromospheric intensity oscillations in these locations. However, for the locations which are away from the flare ribbons, the contribution of the magnetic jerks in driving these oscillations cannot be disregarded. We investigate this further by extracting the approximate estimates of chromospheric velocity amplitudes from $\Delta \mathrm{I} / \mathrm{I}$ of the $\mathrm{H} \alpha$ light-curves assuming that these intensity oscillations are wholly caused by the Doppler shift in the $\mathrm{H} \alpha$ line due to the motion of chromospheric plasma. Under this assumption, the quantity $\Delta \mathrm{I} / \mathrm{I}$ could be the proxy for the chromospheric velocity amplitude. We plot this quantity for all the locations 'K1', 'K2', 'K3', 'K4', 'K5', and 'K6' in the Figure 13. It is seen from these plots that the quantity $\Delta \mathrm{I} / \mathrm{I}$ shows oscillatory behaviour at all the aforementioned locations for the epochs, before and spanning the flare. We do observe some spikes in the temporal behaviour of $\Delta \mathrm{I} / \mathrm{I}$ spanning the flare for the locations ' $\mathrm{K} 2$ ', 'K5', and ' $\mathrm{K} 6$ '. However, it could be attributed to the effect of the flare as these locations are in the vicinity of $\mathrm{H} \alpha$ flare-ribbons. We have also estimated the Fourier power spectrum of the variations in $\Delta \mathrm{I} / \mathrm{I}$ for all the locations ('K1', 'K2', 'K3', 'K4', 'K5', and 'K6') for the pre-flare phase (03:01-06:01 UT) and the flare phase (06:31-09:31 UT) as shown in the Figure 14. It is observed that these power spectra show a general enhancement in the oscillatory power during the flare as compared to the pre-flare phase for all these locations. However, this effect is more for the locations in the vicinity of the $\mathrm{H} \alpha$ flare-loops ('K2', 'K5', and 'K6') as compared to the locations away from the flare-loops ('K1', 'K3', and 'K4'). Thus, our results indicate that these magnetic jerks could also power the chromospheric oscillations, apart from the localized photospheric oscillations.

These magnetically driven oscillations in the active regions are important for understanding the transport of acoustic energy from the photospheric levels to the higher solar atmospheric layers.

Acknowledgements We are thankful to the anonymous referee for constructive comments and suggestions that improved the discussions and presentation of our work in this paper. We acknowledge the use of data 
from HMI instrument on board SDO spacecraft. The $S D O$ is a mission of NASA aimed to understand the causes of solar variability and its impacts on Earth. Our sincere thanks to the HMI team for providing the tracked magnetic and velocity data as per our requirement. This work utilizes the Global Oscillation Network Group (GONG) data obtained by the NSO Integrated Synoptic Program (NISP), managed by the National Solar Observatory, which is operated by AURA, Inc. under a cooperative agreement with the National Science Foundation. Thanks to GONG++ team for providing the processed H $\alpha$ observations. We also acknowledge the use of data from GOES-15 space mission.

\section{References}

Burtseva, O., Martínez-Oliveros, J. C., Petrie, G. J. D., \& Pevtsov, A. A. 2015, ApJ, 806, 173

Domingo, V., Fleck, B., \& Poland, A. I. 1995, Sol. Phys., 162, 1

Fisher, G. H., Canfield, R. C., \& McClymont, A. N. 1985, ApJ, 289, 425

Fisher, G. H., Bercik, D. J., Welsch, B. T., \& Hudson, H. S. 2012, Sol. Phys., 277, 59

Harvey, J. W. 1986, in Small-Scale Magnetic Flux Concentration in the Solar Photosphere, ed. W. Deinzer, M. Knolker, \& H. Voight (Gottingen: Vandenhoeck \& Rupprecht), 25

Harvey, J., \& GONG Instrument Team 1995, GONG 1994. Helio- and Astro-Seismology from the Earth and Space, 76, 432

Harvey, J. W., Hill, F., Hubbard, R. P., et al. 1996, Science, 272, 1284

Harvey, J., Tucker, R., \& Britanik, L. 1998, Structure and Dynamics of the Interior of the Sun and Sun-like Stars, 418, 209

Harvey, J. W., Bolding, J., Clark, R., et al. 2011, Bulletin of the American Astronomical Society, 1745

Hudson, H. S., Fisher, G. H., \& Welsch, B. T. 2008, in ASP Conf. Ser. 383, Subsurface and Atmospheric Influences on Solar Activity, ed. R. Howe et al. (San Francisco, CA: ASP), 221

Kaiser, M. L., Kucera, T. A., Davila, J. M., et al. 2008, Space Sci. Rev., 136, 5

Kosovichev, A. G., \& Zharkova, V. V. 2001, ApJ, 550, L105

Kosugi, T., Matsuzaki, K., Sakao, T., et al. 2007, Sol. Phys., 243, 3

Kumar, B., Venkatakrishnan, P., Mathur, S., Tiwari, S. K., \& García, R. A. 2011, ApJ, 743, 29

Lin, R. P., Dennis, B. R., Hurford, G. J., et al. 2002, Sol. Phys., 210, 3

Nakajima, H., Sekiguchi, H., Sawa, M., Kai, K., \& Kawashima, S. 1985, PASJ, 37, 163

Patterson, A., \& Zirin, H. 1981, ApJ, 243, L99

Patterson, A. 1984, ApJ, 280, 884 
Pesnell, W. D., Thompson, B. J., \& Chamberlin, P. C. 2012, Sol. Phys., 275, 3

Petrie, G. J. D., \& Sudol, J. J. 2010, ApJ, 724, 1218

Petrie, G. J. D. 2014, Sol. Phys., 289, 3663

Press, W. H., Teukolsky, S. A., Vetterling, W. T., \& Flannery, B. P. 1992, Cambridge: University Press, —c1992, 2nd ed.,

Qiu, J., \& Gary, D. E. 2003, ApJ, 599, 615

Scherrer, P. H., Bogart, R. S., Bush, R. I., et al. 1995, Sol. Phys., 162, 129

Sudol, J. J., \& Harvey, J. W. 2005, ApJ, 635, 647

Schou, J., Scherrer, P. H., Bush, R. I., et al. 2012, Sol. Phys., 275, 229

Venkatakrishnan, P., Kumar, B., \& Uddin, W. 2008, MNRAS, 387, L69

Wang, H. 1992, Sol. Phys., 140, 85

Wang, H., Ewell, M. W., Jr., Zirin, H., \& Ai, G. 1994, ApJ, 424, 436

Wang, H., Qiu, J., Denker, C., et al. 2000, ApJ, 542, 1080

Wang, H., Spirock, T. J., Qiu, J., et al. 2002, ApJ, 576, 497 


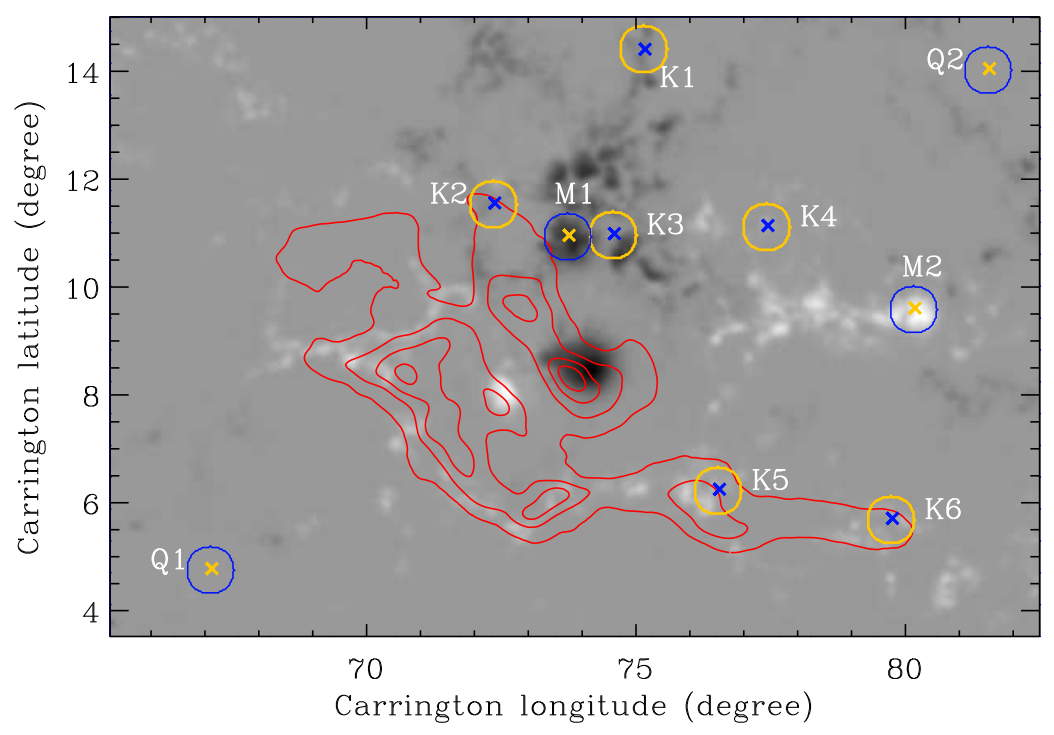

Fig. 4 The background shows the mean line-of-sight photospheric magnetic fields for the period 07:00-08:50 UT on 11 April 2013 in the active region NOAA 11719, as measured in Fe I $6173 \AA$ line by the HMI instrument onboard SDO spacecraft. The overlaid contours shown in red color are the locations of the chromospheric $\mathrm{H} \alpha$ (6562.8 $\AA$ line) flare-ribbons averaged over the period 07:00-08:50 UT on 11 April 2013 in the aforementioned active region during an M6.5 class flare, as observed with the GONG++ instrument. The contours are drawn at the levels $90 \%, 80 \%, 70 \%$ and $60 \%$ of the maximum brightness in $\mathrm{H} \alpha$, from the center of the ribbons, respectively. The crosses shown in blue color within the yellow circles (labelled as 'K1', 'K2', 'K3', 'K4', 'K5', and 'K6') are the centroids of the locations where we observe sudden and persistent changes in the line-of-sight photospheric magnetic fields $\left(B_{l o s}\right)$ in the active region during the flare. Here, it is evident that these locations are in the vicinity of the flare-ribbons and away from the flare-ribbons in the active region. The crosses shown in yellow color within blue circles (labelled as 'M1', 'M2') are the centroids of the locations where we observe gradual evolution of $B_{l o s}$ during the flare whereas the same labelled as 'Q1', and 'Q2' are in the quiet Sun. The field-of-view is $\sim 290 \times 193 \mathrm{arcsec}^{2}$. 

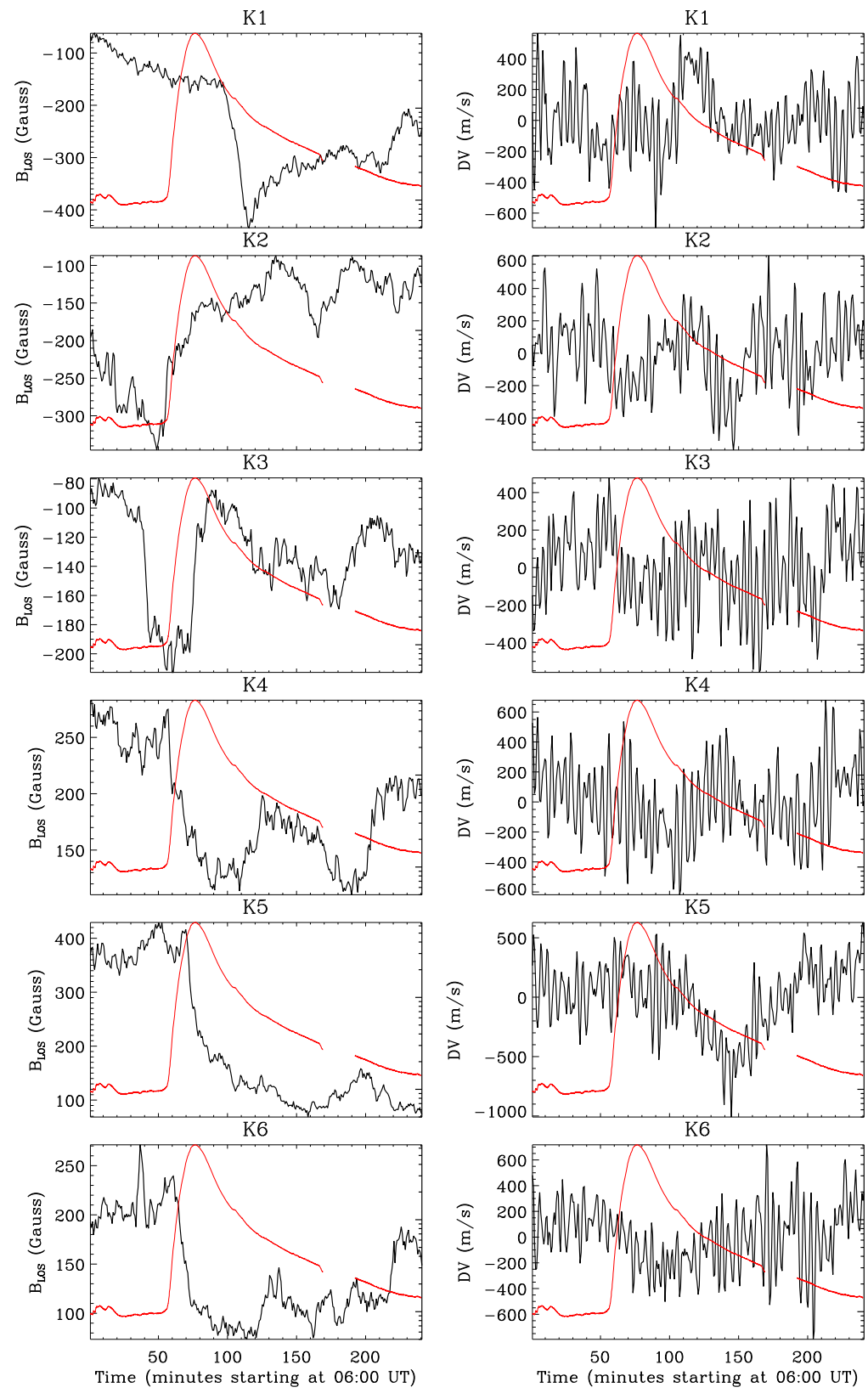

Fig. 5 Plots shown in solid black lines in the left panels represent time evolution of the total lineof-sight photospheric magnetic field $\left(B_{l o s}\right)$ averaged over a raster of nine pixels in the locations 'K1', 'K2', 'K3', 'K4', 'K5', and 'K6' in the active region during the period 06:00-10:00 UT on 11 April 2013 at the cadence of 45 s. Similarly, the plots shown in solid black lines in the right panels represent time evolution of the simultaneous Doppler velocity (DV) in the aforementioned locations after removing the large background gradient due to orbital velocity of the satellite. The $B_{l o s}$ and DV measurements are from HMI. The plots shown in solid red lines represent the evolution of soft X-ray flux in 1-8 $\AA$ energy-band from GOES-15. The $B_{l o s}$ in these locations shows sudden and persistent changes during the flare, which are followed by the perturbations in the DV at these locations. 


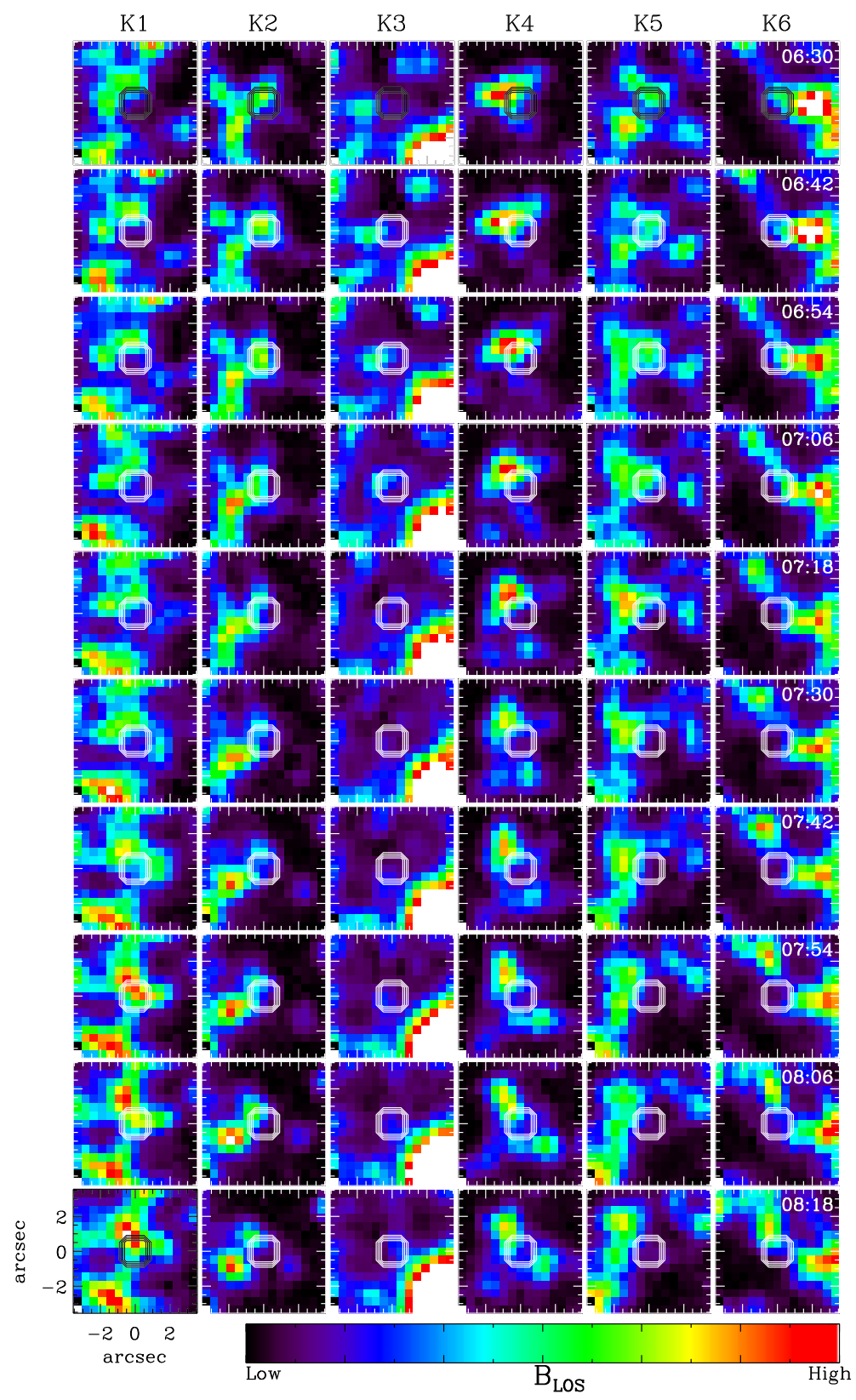

Fig. 6 Mosaic of images showing the time evolution of $B_{l o s}$ over a grid of $15 \times 15$ pixels containing the raster of $3 \times 3$ pixels at its center (enclosed by white box) corresponding to the centroids of the locations 'K1', 'K2', 'K3', 'K4', 'K5', and 'K6' in the active region for the period from 06:30 UT to 08:18 UT at the time interval of twelve minutes. Each column is representing the temporal evolution of $B_{l o s}$ for a given kernel from top to bottom. The dynamic range of $B_{l o s}$ is different for the columns (kernels), however within a column the range is same. For the locations 'K3', and 'K6', the pixels with high values of $B_{l o s}$ are made saturated in order to enhance the visibility of the morphological evolution of magnetic features within the white boxes. The FOV is $\sim 7.5 \times 7.5 \operatorname{arcsec}^{2}$. 

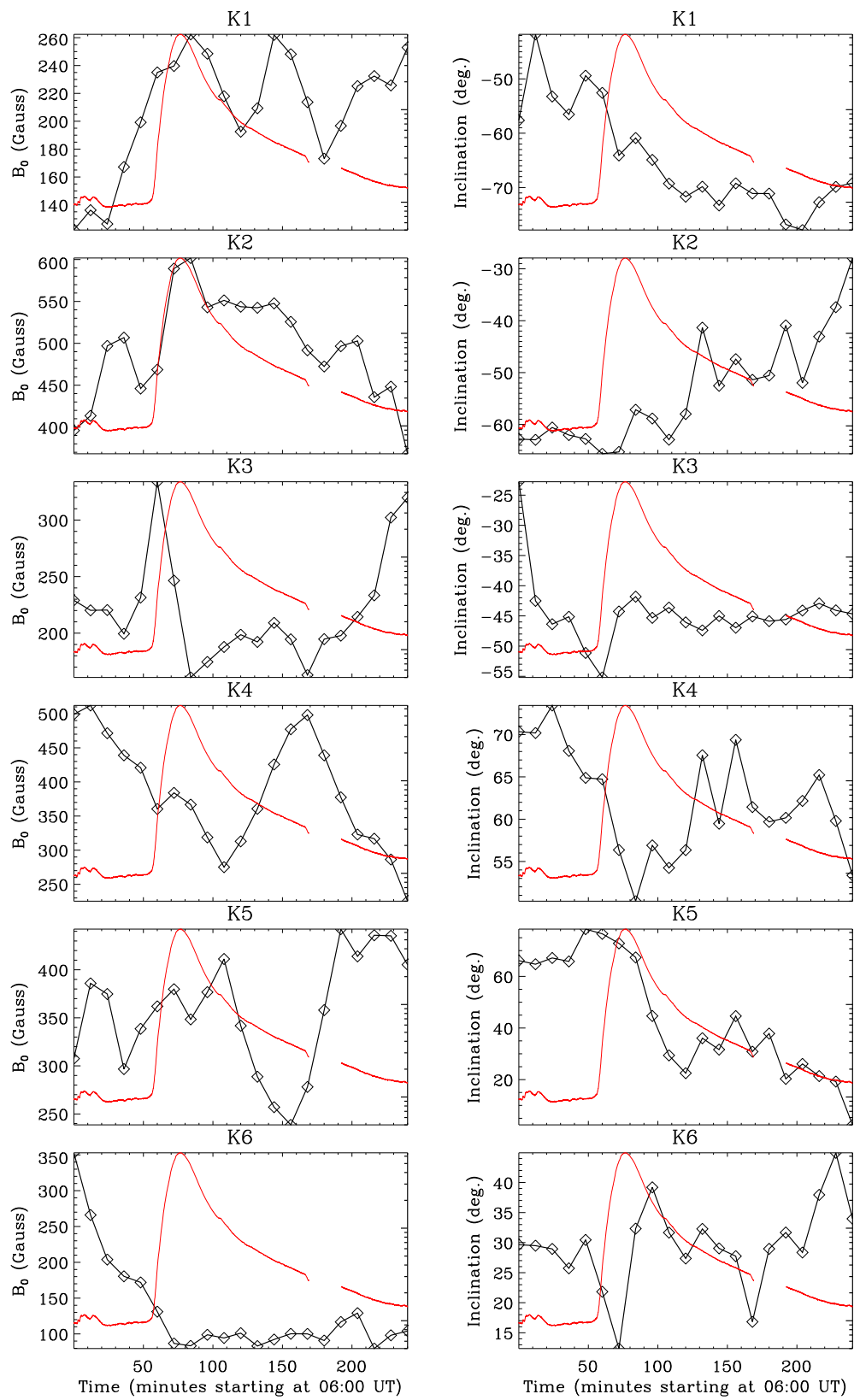

Fig. 7 Plots shown in solid black lines (with diamonds) in the left panels represent time evolution of the total magnetic field strength $\left(B_{0}\right)$ averaged over a raster of nine pixels in the locations 'K1', 'K2', 'K3', 'K4', 'K5', and 'K6' in the active region during the period 06:00-10:00 UT on 11 April 2013 at the cadence of 12 minute. Similarly, the plots shown in solid black lines (with diamonds) in the right panels represent time evolution of the simultaneous inclination angle $(\gamma)$ in the aforementioned locations. The $B_{0}$ and $\gamma$ measurements are from HMI. The plots shown in solid red lines in all the panels represent the evolution of soft X-ray flux in 1-8 $\AA$ energy-band as observed with the GOES-15. Here, we notice that the $B_{0}$ and the $\gamma$, both, show sudden changes in these locations during the flare. 

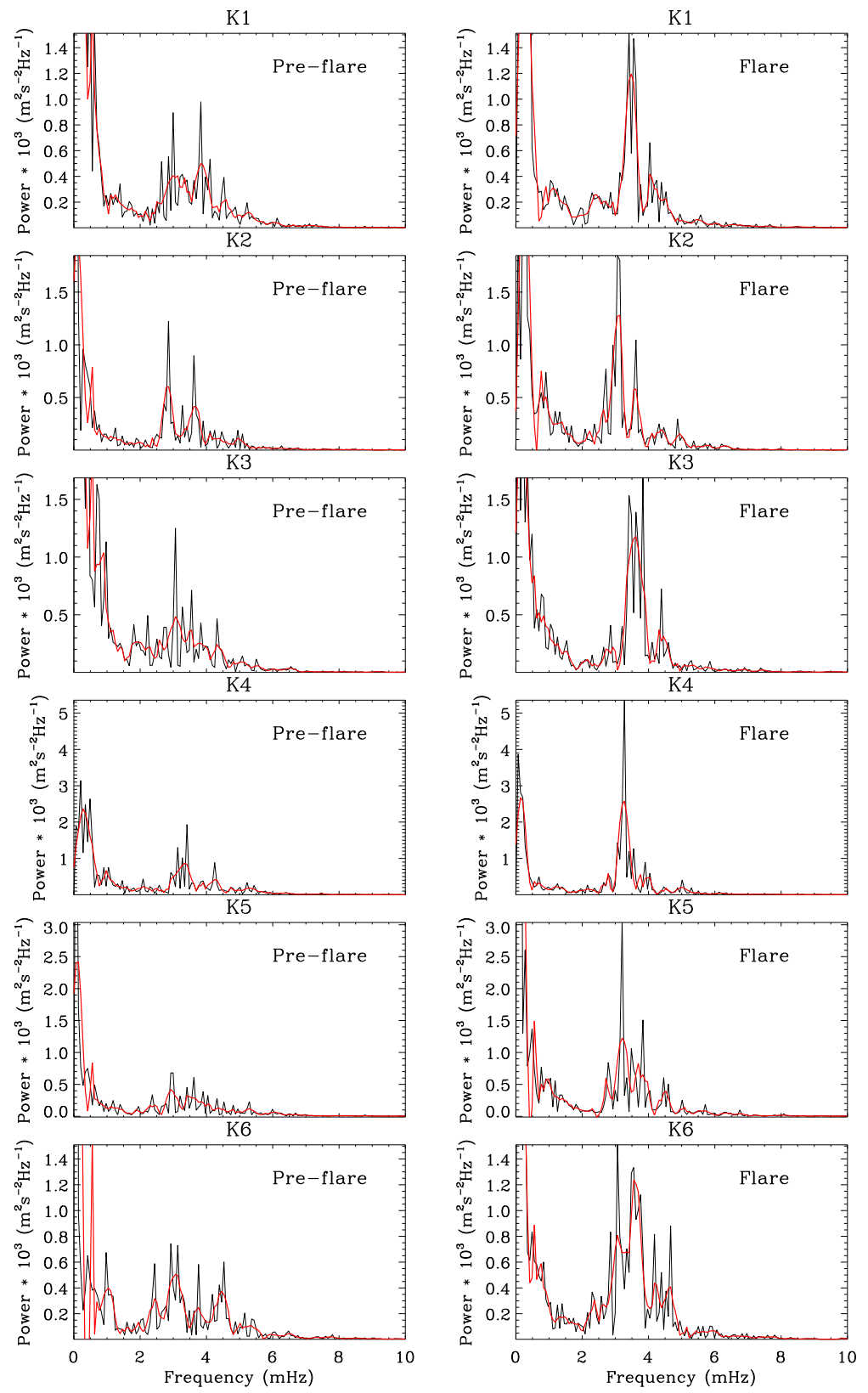

Fig. 8 Plots shown in solid black lines in the left panels represent the average Fourier power spectrum of velocity oscillations estimated over a raster of nine pixels in the locations ' $\mathrm{K} 1$ ', 'K2', 'K3', 'K4', 'K5', and 'K6' in the active region during the period 01:00-05:00 UT on 11 April 2013. Similarly, the plots shown in solid black lines in the right panels represent the average Fourier power spectrum of velocity oscillations estimated over the same rasters of nine pixels in the aforementioned locations during the period 06:00-10:00 UT on 11 April 2013. The plots shown in solid red lines in all the panels represent a smoothing fit (Savitszky-Golay Fit) applied to the original power spectrum to estimate the power envelopes. These plots show that the acoustic power is enhanced in the aforementioned locations in the $2-5 \mathrm{mHz}$ frequency band during the flare. 

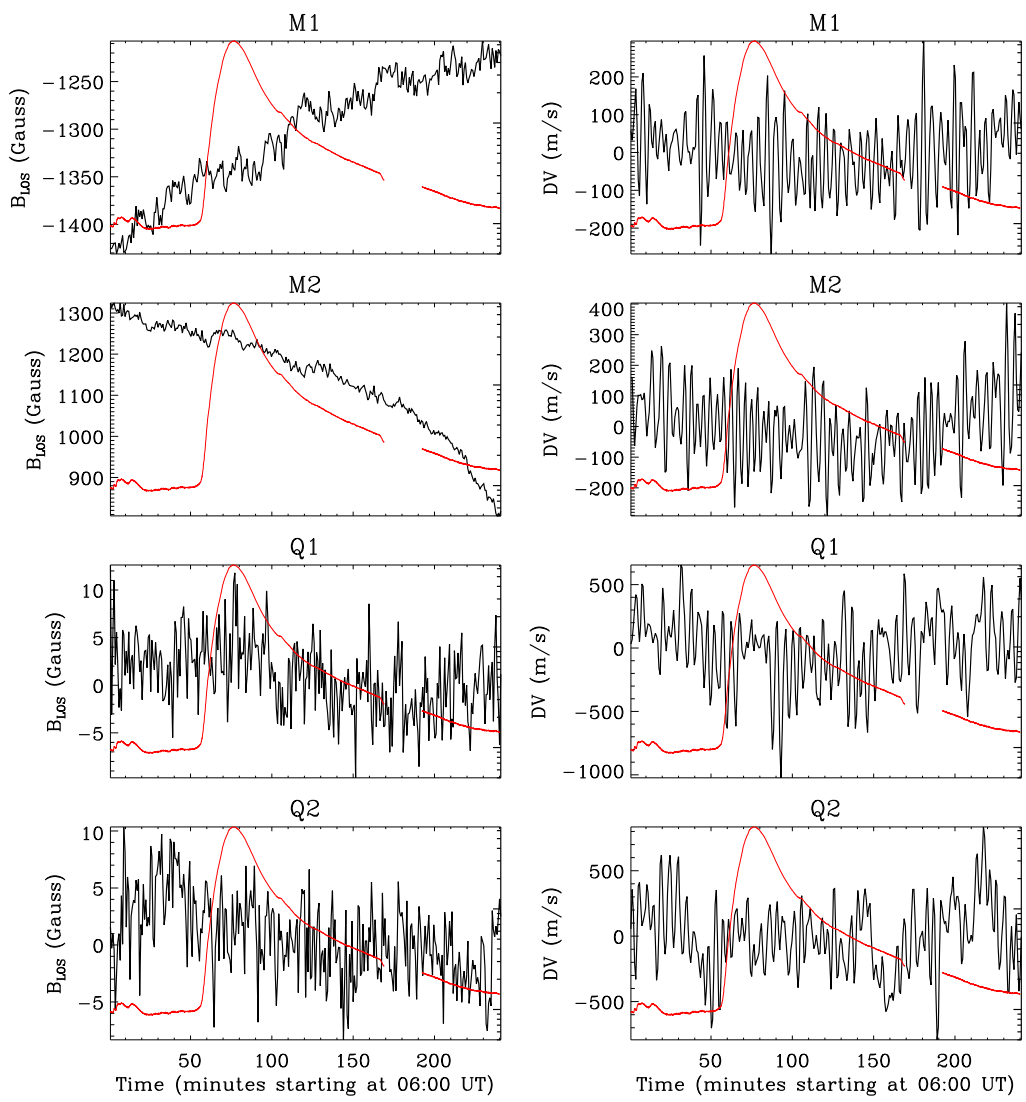

Fig.9 Same as Figure 5, but for the locations 'M1', and 'M2' in the active region and the locations 'Q1', and 'Q2' in the quiet Sun. Here, we notice that the line-of-sight photospheric magnetic fields $\left(B_{l o s}\right)$ show gradual evolution in the locations 'M1', and 'M2' during the flare. We do not observe any significant changes in the photospheric Doppler velocity (DV) in these locations during the flare. In the quiet locations 'Q1', and 'Q2', the variations in the mean $B_{l o s}$ are within the noise level of the instrument $(\sim 10 \mathrm{G})$ while the DV is dominant and shows normal evolution during the flare. 

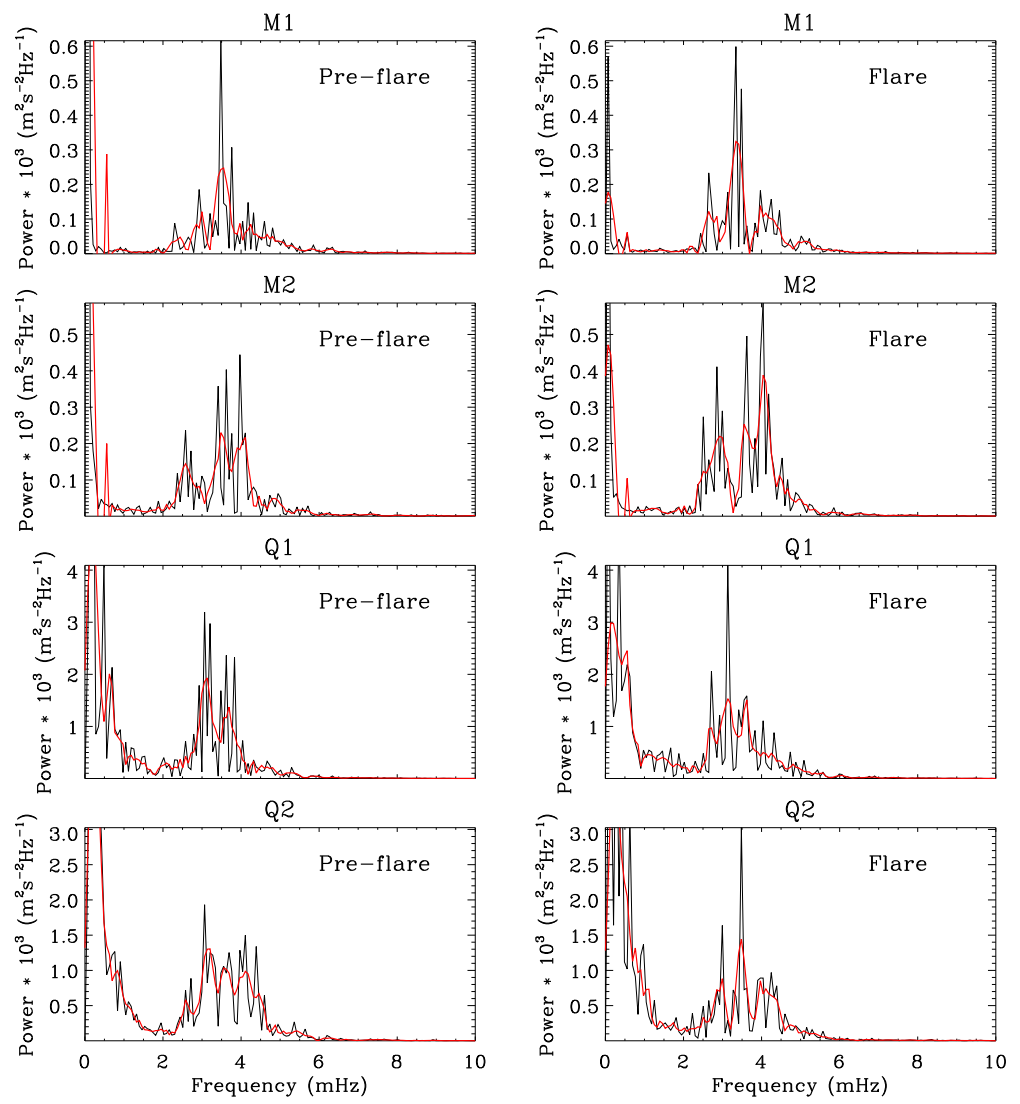

Fig. 10 Same as Figure 8, but for the locations 'M1', and 'M2', in the active region and the locations 'Q1', and 'Q2' in the quiet Sun. Here, we notice that the power of velocity oscillations is enhanced in the locations 'M1', and 'M2' in the $2-5 \mathrm{mHz}$ frequency band during the flare. This enhancement in power is more for 'M2' as compared to that for 'M1' which could be attributed to the amount of reduction in the line-of-sight photospheric magnetic fields in the active region during the flare. In the case of 'Q1', and 'Q2', we do not observe any significant variations in the power of velocity oscillations during the flare, being in the quiet Sun. 

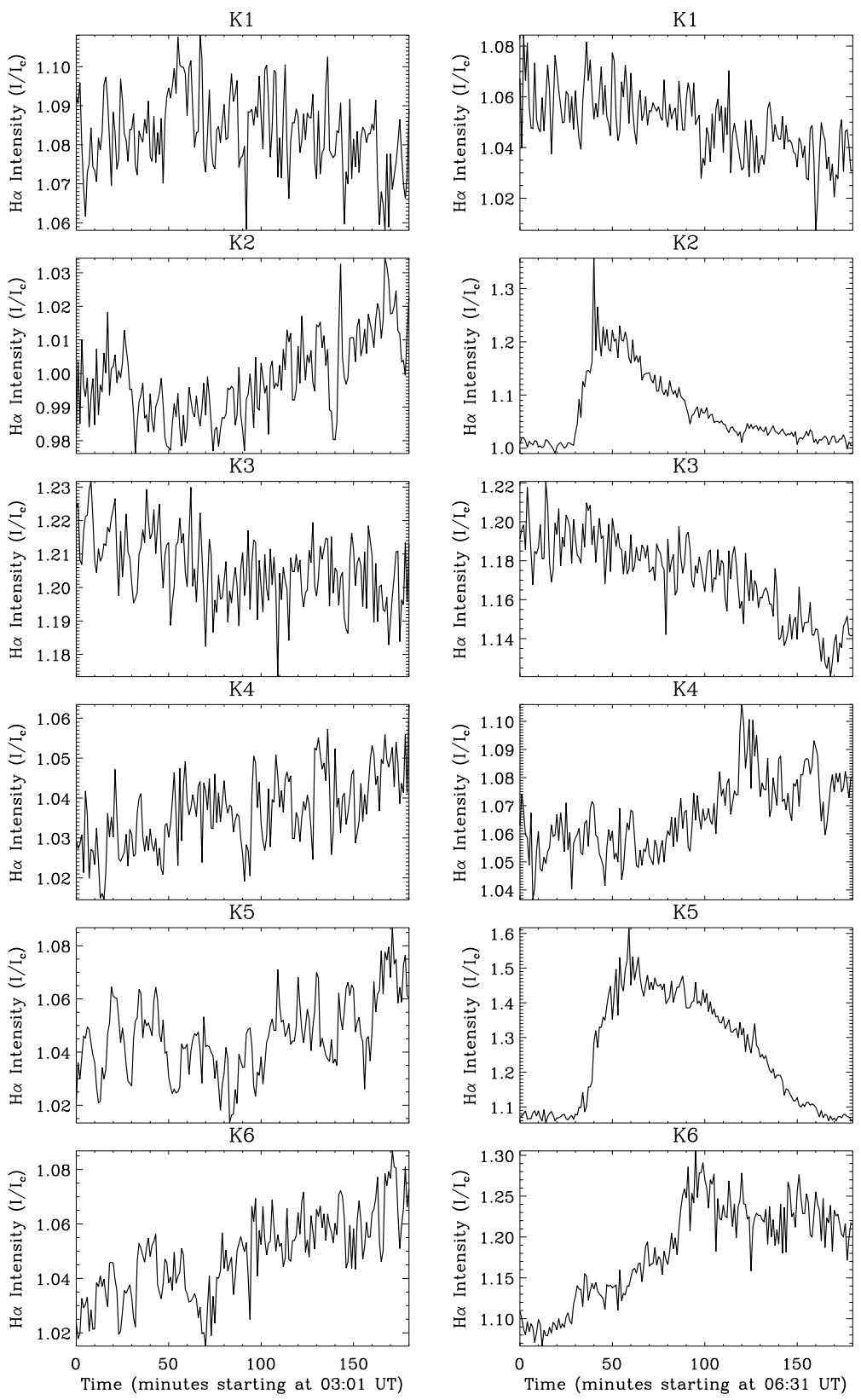

Fig. 11 Plots shown in solid black lines in the left panels represent the time evolution of normalized $\mathrm{H} \alpha$ light-curves averaged over a raster of nine pixels within the locations ' $\mathrm{K} 1$ ', 'K2', 'K3', 'K4', 'K5', and 'K6' in the active region during the period 03:01-06:01 UT on 11 April 2013 (pre-flare condition). Similarly, the plots shown in solid black lines in the right panels represent the time evolution of normalized $\mathrm{H} \alpha$ light-curves averaged over the same rasters of nine pixels within the aformentioned locations during the period 06:31-09:31 UT on 11 April 2013 (spanning the flare). Here, the observational cadence is one minute. 

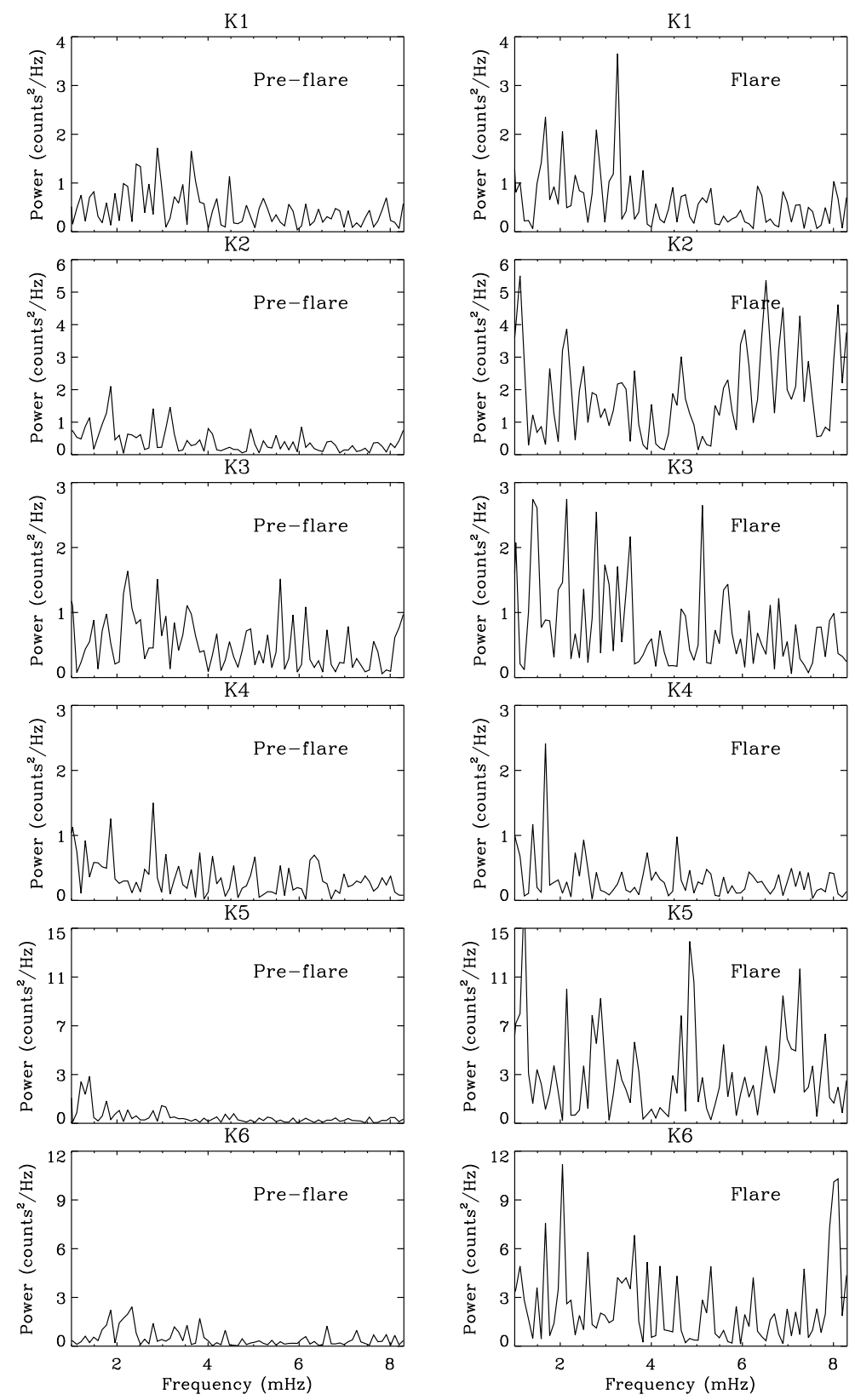

Fig. 12 Plots shown in solid black lines in the left panels show the average Fourier power spectrum of $\mathrm{H} \alpha$ intensity oscillations estimated over a raster of nine pixels within the locations ' $\mathrm{K} 1$ ', 'K2', 'K3', 'K4', 'K5', and 'K6' in the active region during the period 03:01-06:01 UT on 11 April 2013 (pre-flare condition). Similarly, the plots shown in solid black lines in the right panels show the average Fourier power spectrum of $\mathrm{H} \alpha$ intensity oscillations estimated over the same rasters of nine pixels within the aforementioned locations of the active region for the period 06:31-09:31 UT on 11 April 2013 (spanning the flare). It is evident that the power of $\mathrm{H} \alpha$ intensity oscillations is enhanced in the aforementioned locations during the flare. 

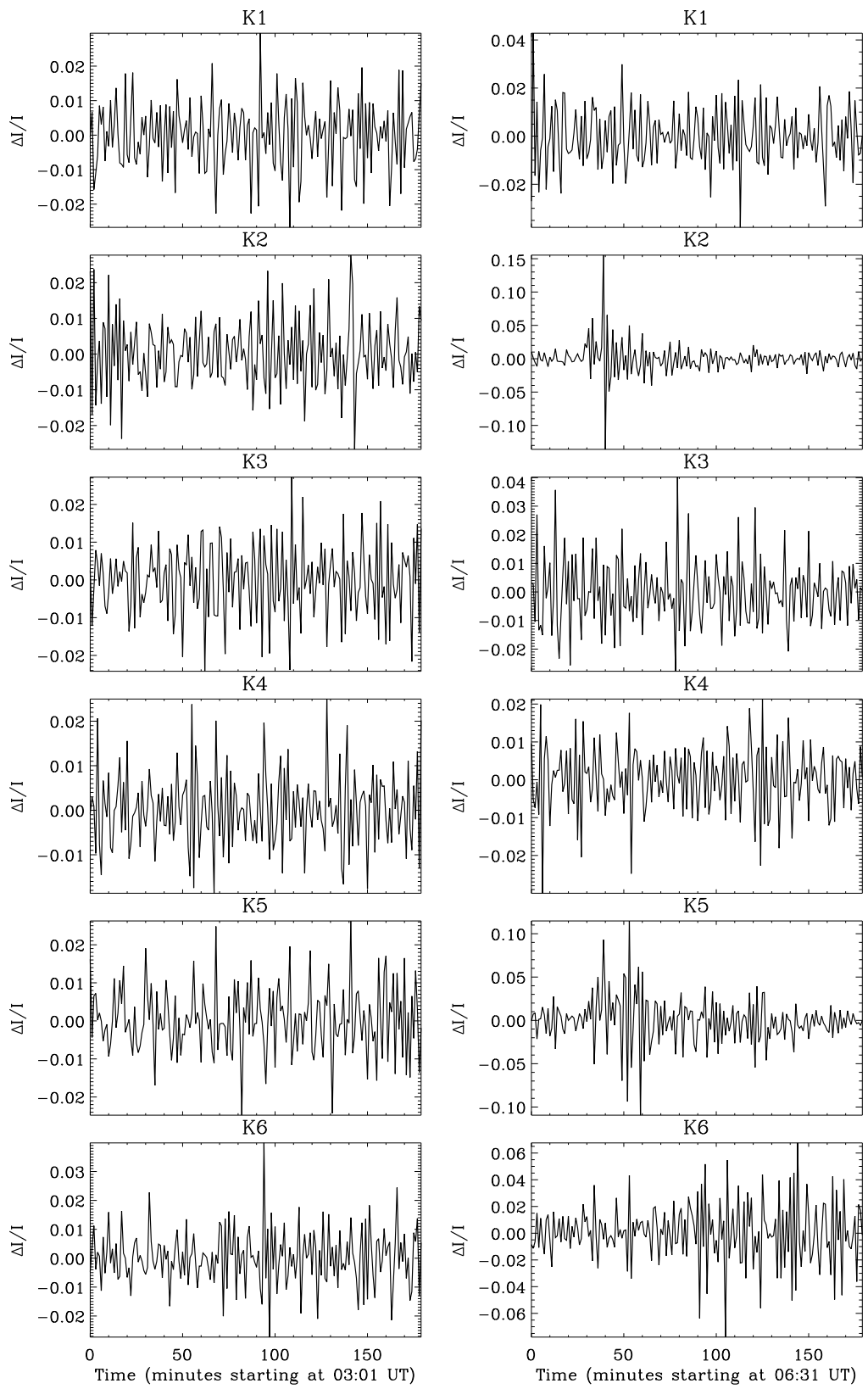

Fig. 13 Plots shown in solid black lines in the left panels represent time evolution of $\Delta \mathrm{I} / \mathrm{I}$ (extracted from $\mathrm{H} \alpha$ light-curves) averaged over a raster of nine pixels within the locations ' $\mathrm{K} 1$ ', 'K2', 'K3', 'K4', 'K5', and 'K6' in the active region during the period 03:01-06:01 UT on 11 April 2013 (pre-flare condition). Similarly, the plots shown in solid black lines in the right panels represent the time evolution of $\Delta \mathrm{I} / \mathrm{I}$ (extracted from $\mathrm{H} \alpha$ light-curves) averaged over the same rasters of nine pixels within the aformentioned locations during the period 06:31-09:31 UT on 11 April 2013 (spanning the flare). The oscillatory behaviour of the quantity $\Delta \mathrm{I} / \mathrm{I}$ (proxy for chromospheric velocity amplitude) is clearly seen in these plots. 

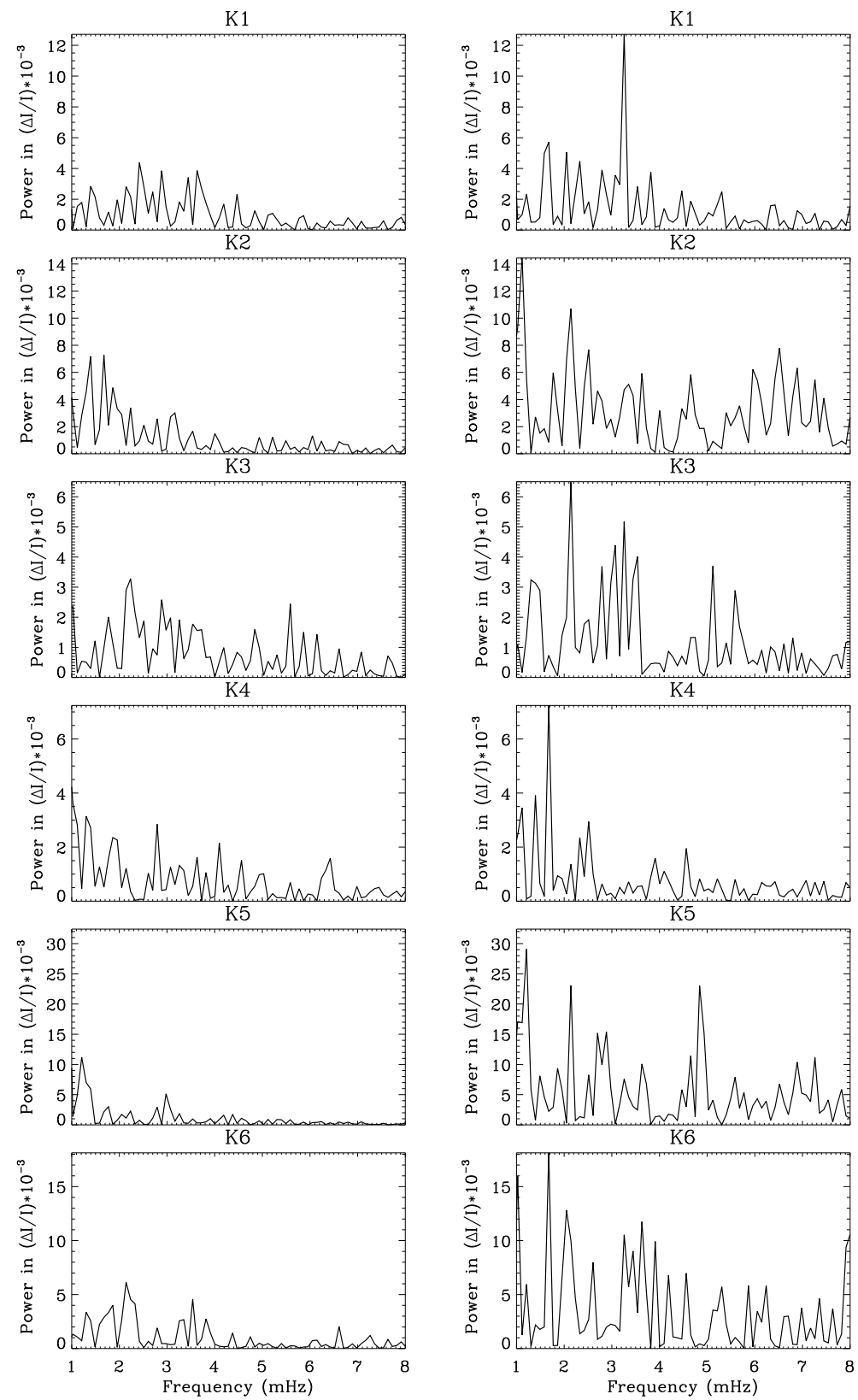

Fig. 14 Plots shown in solid black lines in the left panels show the average Fourier power spectrum of $\Delta \mathrm{I} / \mathrm{I}$ (extracted from $\mathrm{H} \alpha$ light-curves) estimated over a raster of nine pixels within the locations 'K1', 'K2', 'K3', 'K4', 'K5', and 'K6' in the active region during the period 03:0106:01 UT on 11 April 2013 (pre-flare condition). Similarly, the plots shown in solid black lines in the right panels show the average Fourier power spectrum of $\Delta \mathrm{I} / \mathrm{I}$ (extracted from $\mathrm{H} \alpha$ lightcurves) estimated over the same rasters of nine pixels within the aforementioned locations of the active region for the period 06:31-09:31 UT on 11 April 2013 (spanning the flare). It is evident that the power of $\Delta \mathrm{I} / \mathrm{I}$ oscillations (proxy for chromospheic velocity oscillations) is enhanced in the aforementioned locations during the flare. 